\title{
Integration of Reactive, Torque-Based Self-Collision Avoidance Into a Task Hierarchy
}

\author{
Alexander Dietrich, Thomas Wimböck, Alin Albu-Schäffer, and Gerd Hirzinger
}

\begin{abstract}
Reactively dealing with self-collisions is an important requirement on multi-DOF robots in unstructured and dynamic environments. Classical methods to integrate respective algorithms into task hierarchies cause substantial problems: Either these unilateral safety constraints are permanently active, unnecessarily locking DOF for other tasks, or they get activated online and result in a discontinuous control law. We propose a new, reactive self-collision avoidance algorithm for highly complex robotic systems with a large number of DOF. In particular, configuration dependent damping is imposed to dissipate undesired kinetic energy in a well-directed manner. Moreover, we merge the algorithm with a novel method to incorporate these unilateral constraints into a dynamic task hierarchy. Our approach both allows to specifically limit the force/torque derivative to comply with physical constraints of the real robot and to prevent discontinuities in the control law while activating/deactivating the constraints. No redundancy is wasted. No comparable algorithms have been developed and implemented on a torque controlled robot with such a level of complexity so far. The implementation of our generic solution on the multi-DOF humanoid Justin clearly validates the performance and demonstrates the real-time applicability of our synthetic approach. The proposed method can be used to contribute to whole-body controllers.
\end{abstract}

Index Terms-Self-Collision Avoidance, Task Hierarchy, Force Control, Redundant Robots

\section{INTRODUCTION}

Establishing robots in domestic environments requires them to be both compliant and versatile. Compliance can be achieved by equipping the manipulator with sufficient force/torque sensing capabilities so as to allow the system to "feel" contacts and interact with the environment or by applying intrinsically compliant joints [1]. Versatility, amongst others, can be realized by providing many independently controllable degrees of freedom (DOF) which make it possible to execute several tasks and comply with multiple physical constraints simultaneously. Up to the present, several robotic systems have been developed in this respect [2], [3], [4], [5].

Nevertheless, a multi-DOF structure significantly increases the complexity in terms of whole-body coordination. A crucial aspect is the treatment of (self-)collision situations, see Fig. 1. Using only planning algorithms to avoid collisions is not sufficient if considering manipulators in unstructured and dynamic environments. The robot must be enabled to detect critical situations and react in real-time [6], [7], [8], [9], [10]. In [11], Sugiura et al. propose to generate repulsive forces between potentially colliding body links and transform them into corresponding joint motions in order to access the velocity

The authors are with the German Aerospace Center (DLR), Institute of Robotics and Mechatronics, Wessling D-82230, Germany; (Contact: alexander.dietrich@dlr.de, thomas.wimboeck@dlr.de, alin.albuschaeffer@dlr.de, gerd.hirzinger@dlr.de)

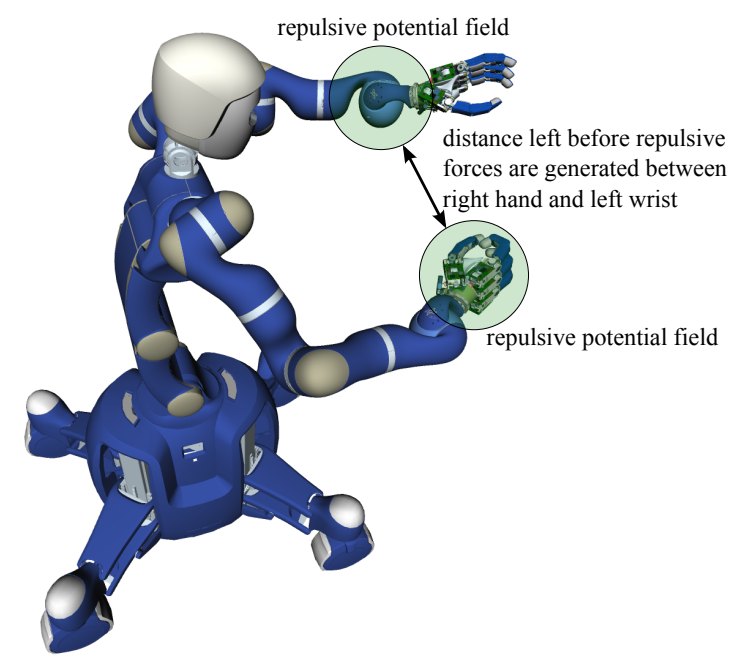

Fig. 1. Unilateral constraints (repulsive potential fields) are utilized to avoid self-collisions, exemplified on the humanoid robot Justin of the German Aerospace Center (DLR).

control interface. Null space projection techniques [12], [13], [14] are utilized in that whole-body control framework. Using repulsive potential fields [6], introduced by Khatib, to achieve collision avoidance is a well-established approach by now. However, combining these unilateral constraints with classical null space projection concepts causes a great problem. Usually, one should settle such safety features at the top levels within the priority order. When switching between inactive and active repulsive potential fields, a discontinuity in the null space projector occurs while using classical approaches, leading to a discontinuous control law. One solution is to "lock" the respective directions so as to prevent the discontinuity. However, that is equal to a waste of the structural redundancy in noncritical configurations. The already mentioned framework [11] integrates a self-collision avoidance into a task hierarchy. Concerning the collision avoidance interventions, damping is not regarded. But dissipating kinetic energy becomes crucial when considering more than one potential collision simultaneously. The irregularities caused by the unilateral constraints are also not considered yet. In [15], Ellekilde and Christensen use a dynamical approach to scale task contributions online. Still, competitive dynamics result and the redundancy is not optimally exploited. Brock et al. propose a dynamic hierarchy in [16]. Herein, obstacle avoidance is realized in the null space of another task. It is given a higher priority if that null space reveals to be not sufficient to ensure a collisionfree motion. A suitable coefficient is calculated online and induces the transition. However, the range for the coefficient has to be determined experimentally and the approach requires 
user-defined time windows to specify the transient behavior. In [17], Lee et al. smooth the transition while using modified null space projection techniques. The framework bases on considerations in the kinematic domain where joint velocities are inputs to the robot. As good compliance calls for force/torque control instead of stiff position/velocity control, the approach is not applicable for our purposes. Seminal work has been done by Mansard et al. [18]. The work introduces a new inversion operator for the computation of appropriate null spaces and applies the continuous control law to a visual servoing scenario. In [19], an extension for a hierarchy of tasks and unilateral constraints is made which is based on [20]. However, a specific adaption to the physical constraints of force/torque controlled systems, i. e., the limits of the force/torque control loops, is not provided. Several other established solutions are optimization-based [21], [22]. In [22], inequality constraints are incorporated into an optimization problem formulation. In a kinematic example, the activation process is smoothed by adding constraints to higher derivatives of the system output. In [21], a weighting of different constraints has to be performed and an activation coefficient is introduced. Furthermore, there exist various established concepts to smooth irregularities by utilizing damped least-squares techniques for the inversion process. Deo and Walker provide an overview in [23]. These approaches are widely used in inverse kinematics [24], [25], [26], [27], [20]. These methods offer an easy way to smooth discontinuities but the parameterization of the damping values is not intuitive since the direct relation to physical values of the real system is not given. Last we want to mention iterative methods to deal with that redundancy issue. Raunhardt and Boulic introduce temporary joint limit constraints in an iterative inverse kinematics algorithm in [28]. This kind of method is suitable for 3D characters or virtual mannequins. However, using iterative approaches is problematic if hard real-time matters. The complexity of a self-collision avoidance poses difficulties in terms of nondeterminism of the redundancy resolution algorithm. Thus, such methods are rarely applied on real robots.

In this paper, a new redundancy resolution method is provided and a self-collision avoidance is integrated into the obtained dynamic task hierarchy. The goal is to achieve an order of various, prioritized tasks such that more important tasks are fulfilled while less important ones are not necessarily completed. The concept is based on our recent works [29], [30] and combines them to a novel framework. Our algorithm for reactive self-collision avoidance [29] extends the work initiated by De Santis et al. in [9]. Compared to the majority of the state-of-the-art approaches, we do not remain on the kinematic level but we address the dynamic domain where forces and torques are the inputs of the robot. Our concept is a generic solution to the problem of self-collisions which comprises a configuration dependent damping design for systematic dissipation of undesired kinetic energy in a welldirected manner. The algorithm allows to incorporate a large number of potentially colliding body segments simultaneously while still remaining feasible in real-time ${ }^{1}$. Being unilateral,

\footnotetext{
${ }^{1}$ Here, the algorithm is implemented in a $1 \mathrm{~ms}$ control cycle.
}

these safety features call for special treatment concerning their insertion into a task hierarchy. In this context, we extend classical null space projection methods by transition shaping to account for that special property [30]. We derive the theoretical framework which allows to specifically limit the force/torque derivative during the activation/deactivation process of potential fields. By means of that we close the gap between the abstract mathematical structure of a task hierarchy and the influence on real physical values in the robotic system. We prove that only the behavior in the direction of the unilateral constraint is altered by the transition shaping. Both self-collision avoidance and redundancy resolution are merged and implemented on a torque controlled, multi-DOF humanoid. There do not exist comparable approaches and implementations in terms of complexity and performance so far. Several experiments validate our concept and demonstrate the real-time applicability of our synthetic approach.

The work is organized as follows. At first we derive the self-collision avoidance algorithm in Sec. II. Experiments are conducted to verify its performance. Sec. III focuses on the redundancy resolution concept to incorporate unilateral constraints in a dynamic task hierarchy. Simulations on a simple 3 DOF system are presented. Sec. IV describes the fusion of both concepts and the implementation on a real robotic system. Sec. V compares our approach with state-ofthe-art techniques and hints at limitations. Experiments on the humanoid Justin are performed and analyzed in Sec. VI.

\section{Self-Collision Avoidance}

As a basic prerequisite for our self-collision avoidance approach which utilizes artificial repulsive potential fields [6], we require information about potentially colliding body links in real-time. That includes $n_{\mathrm{p}}$ contact point pairs with minimum distance and a specification of the robot links they are placed on. In this respect, we adapted the well-established formulation by Gilbert et al. [31] for the geometric collision model of the manipulator. Throughout the rest of this work, we use the word joint torque in place of force/torque since the majority of the robotic systems is rather equipped with revolute than prismatic joints.

First, we define the torque command $\tau_{\text {coll }} \in \mathbb{R}^{n}$ for selfcollision avoidance on an $n$ DOF manipulator:

$$
\boldsymbol{\tau}_{\mathrm{coll}}=-\left(\frac{\partial V_{\mathrm{rep}, \mathrm{tot}}(\boldsymbol{q})}{\partial \boldsymbol{q}}\right)^{T}-\boldsymbol{D}(\boldsymbol{q}) \dot{\boldsymbol{q}},
$$

where $V_{\text {rep,tot }}(\boldsymbol{q}) \in \mathbb{R}_{0}^{+}$expresses the total potential energy of all $n_{\mathrm{p}}$ repulsive potential fields applied to the geometric model of the robot. The configuration of the system is defined by the joint vector $\boldsymbol{q} \in \mathbb{R}^{n}$. Each potential refers to the contact point pair defined by point $i$ and corresponding point $j$ with the assignment $h: i \mapsto j \forall i=1 \ldots n_{\mathrm{p}}$ and is denoted as

$$
V_{\mathrm{rep}, \mathrm{tot}}(\boldsymbol{q})=\sum_{i=1}^{n_{\mathrm{p}}} V_{\mathrm{rep}, i, h(i)}(\boldsymbol{q}) .
$$

In (1), additional damping is injected through the positive definite matrix $\boldsymbol{D}(\boldsymbol{q}) \in \mathbb{R}^{n \times n}$. That configuration dependent term for energy dissipation will be derived in a later section. 


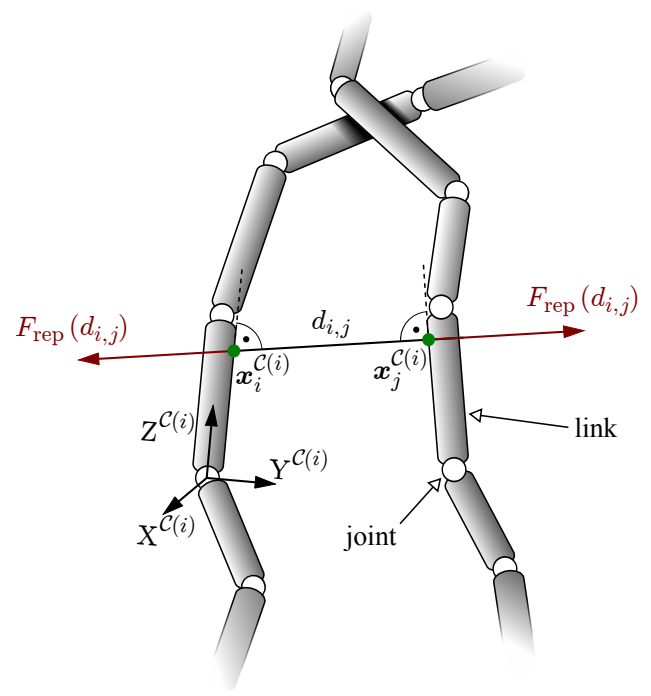

Fig. 2. Relations between two arbitrary contact points $\boldsymbol{x}_{i}^{\mathcal{C}(i)}$ and $\boldsymbol{x}_{j}^{\mathcal{C}(i)}$ and repulsive forces $F_{\text {rep }}\left(d_{i, j}\right)$. The figure illustrates a 3-dimensional example.

\section{A. Repulsive Effect}

For the further analysis, the distance $d_{i, j} \in \mathbb{R}_{0}^{+}$between two contact points $\boldsymbol{x}_{i}, \boldsymbol{x}_{j} \in \mathbb{R}^{3}$ is defined as

$$
d_{i, j}=\left\|\boldsymbol{x}_{i}^{\mathcal{C}(i)}-\boldsymbol{x}_{j}^{\mathcal{C}(i)}\right\|=\left\|\boldsymbol{x}_{j}^{\mathcal{C}(j)}-\boldsymbol{x}_{i}^{\mathcal{C}(j)}\right\| .
$$

The superscript describes the coordinate frame of the link the indicated contact point lies on. Fig. 2 illustrates an arbitrary contact point pair in a 3-dimensional example. The repulsive forces $F_{\text {rep }}\left(d_{i, j}\right) \in \mathbb{R}_{0}^{+}$are perpendicular to the surfaces of the links and indicate in the directions $\pm \boldsymbol{e}_{i}$ :

$$
\boldsymbol{e}_{i}=\frac{\boldsymbol{x}_{j}^{\mathcal{C}(i)}-\boldsymbol{x}_{i}^{\mathcal{C}(i)}}{d_{i, j}} .
$$

As of now, the superscript will be omitted in the notations. In Fig. 2 it is indicated that the repulsive effect can be described as a function of the distance $d_{i, j}$ between two proximate points $\boldsymbol{x}_{i}$ and $\boldsymbol{x}_{j}$ on different links.

$$
\begin{aligned}
\frac{\partial V_{\mathrm{rep}, i, j}(\boldsymbol{q})}{\partial \boldsymbol{q}} & =\frac{\partial V_{\mathrm{rep}, i, j}}{\partial d_{i, j}} \frac{\partial d_{i, j}}{\partial\left(\boldsymbol{x}_{i}^{T}, \boldsymbol{x}_{j}^{T}\right)^{T}} \frac{\partial\left(\boldsymbol{x}_{i}^{T}, \boldsymbol{x}_{j}^{T}\right)^{T}}{\partial \boldsymbol{q}} \\
& =\frac{\partial V_{\mathrm{rep}, i, j}}{\partial d_{i, j}}\left(\frac{\partial d_{i, j}}{\partial \boldsymbol{x}_{i}} \frac{\partial d_{i, j}}{\partial \boldsymbol{x}_{j}}\right)\left(\begin{array}{ll}
\frac{\partial \boldsymbol{x}_{i}}{\partial \boldsymbol{q}_{i}} & \frac{\partial \boldsymbol{x}_{i}}{\partial \boldsymbol{q}_{j}} \\
\frac{\partial \boldsymbol{x}_{j}}{\partial \boldsymbol{q}_{i}} & \frac{\partial \boldsymbol{x}_{j}}{\partial \boldsymbol{q}_{j}}
\end{array}\right) .
\end{aligned}
$$

Herein, vectors $\boldsymbol{q}_{i}$ and $\boldsymbol{q}_{j}$ denote the joint values which directly ${ }^{2}$ affect the location of $\boldsymbol{x}_{i}$ and $\boldsymbol{x}_{j}$, respectively. In this example, $\boldsymbol{q}_{i}$ and $\boldsymbol{q}_{j}$ describe the joint positions of the left and the right manipulator, respectively. Notice that in general the contact points may have the same base of the kinematic chain so that $\boldsymbol{q}_{i}$ and $\boldsymbol{q}_{j}$ have an intersection. However, just the joints after the branch-off point are relevant. It follows from the multiplication of $\partial d_{i, j} / \partial\left(\boldsymbol{x}_{i}^{T}, \boldsymbol{x}_{j}^{T}\right)^{T}$ and $\partial\left(\boldsymbol{x}_{i}^{T}, \boldsymbol{x}_{j}^{T}\right)^{T} / \partial \boldsymbol{q}$

\footnotetext{
${ }^{2}$ Indirect influence implies the point's motion on the surface of the link due to the motion of the corresponding contact point partner.
}

that just the principal block diagonal of $\partial\left(\boldsymbol{x}_{i}^{T}, \boldsymbol{x}_{j}^{T}\right)^{T} / \partial \boldsymbol{q}$ has influence on the result. The residual multiplications result in zero since the factors are always orthogonal. As an example, let us consider $\partial d_{i, j} / \partial \boldsymbol{x}_{j}$ and $\partial \boldsymbol{x}_{j} / \partial \boldsymbol{q}_{i}$ in the context of Fig. 2. The direction of $\partial d_{i, j} / \partial \boldsymbol{x}_{j}$ is orthogonal to the surface of the link on which $\boldsymbol{x}_{j}$ is lying, whereas $\boldsymbol{q}_{i}$ is only able to let $\boldsymbol{x}_{j}$ move on this surface (indirect influence). Hence, (5) can be simplified to

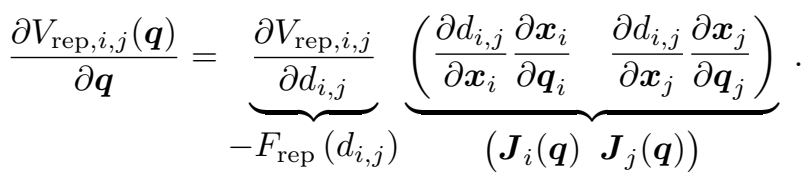

Notice that the right part describes the projection of the force $F_{\text {rep }}\left(d_{i, j}\right)$ into joint space, i. e., the Jacobian matrices $\boldsymbol{J}_{i}(\boldsymbol{q})$ and $\boldsymbol{J}_{j}(\boldsymbol{q})$, respectively. Further usage of these mappings will be in the damping design section.

The repulsive potentials $V_{\text {rep }, i, j}(\boldsymbol{q})$ are supposed to be zero at a specified distance $d_{i, j}=d_{0}$. This is due to the requirement of the collision avoidance being a unilateral constraint. If $d_{0}$ is exceeded, no torque shall be applied. We propose the following piecewise defined function:

$$
V_{\text {rep }, i, j}\left(d_{i, j}\right)=\left\{\begin{array}{ll}
-\frac{F_{\max }}{3 d_{0}^{2}}\left(d_{i, j}-d_{0}\right)^{3} & \forall d_{i, j} \leq d_{0} \\
0 & \forall d_{i, j}>d_{0}
\end{array} .\right.
$$

Here, the potential $V_{\mathrm{rep}, i, j}\left(d_{i, j}\right)$ is of type $C^{2}$, whereas the corresponding repulsive force

$$
\begin{aligned}
F_{\text {rep }}\left(d_{i, j}\right) & =-\frac{\partial V_{\mathrm{rep}, i, j}\left(d_{i, j}\right)}{\partial d_{i, j}} \\
& = \begin{cases}\frac{F_{\max }}{d_{0}^{2}}\left(d_{i, j}-d_{0}\right)^{2} & \forall d_{i, j} \leq d_{0} \\
0 & \forall d_{i, j}>d_{0}\end{cases}
\end{aligned}
$$

is a $C^{1}$ function w.r.t. $d_{i, j}$. An additional design parameter, namely the maximum force $F_{\max } \in \mathbb{R}^{+}$, must be specified in (7) or (8), respectively. To parametrize the fields, the maximum local stiffness $\left.\left(\partial F_{\text {rep }}\left(d_{i, j}\right) / \partial d_{i, j}\right)\right|_{d_{i, j}=0}$ can be limited to a feasible value which is determined by the sample time of the controller. Since we have multiple contact point pairs and configuration dependent relations between force and joint torques, the design is not easy in general. However, a rough estimation for the worst case $F_{\max }$, i.e. one contact point pair for a collision endangered configuration, can be made [29].

The damping force which will be derived in the next section is directly dependent on the local potential stiffness, i.e., $\partial^{2} V_{\text {rep }, i, j}\left(d_{i, j}\right) / \partial d_{i, j}^{2}$. To ensure a continuous control law, $V_{\text {rep }, i, j}\left(d_{i, j}\right)$ must at least be of type $C^{2}$.

\section{B. Damping Design}

In the following we propose a damping design for systematic energy dissipation. The method will allow to specify a damping ratio $\zeta$ to a contact point pair. Therefore, we have to transform the dynamical equations into the operational space [12] which is defined by the collision directions.

In consequence of the configuration dependence we must take the system inertia distribution into account. We define $\dot{d}_{i}$ 


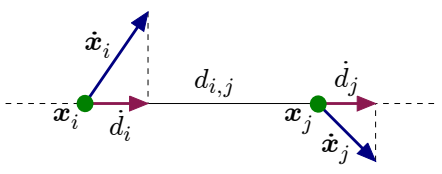

Fig. 3. Illustration of the projected motions of $\boldsymbol{x}_{i}$ and $\boldsymbol{x}_{j}$ into the direction of the collision. Positive directions are defined from $\boldsymbol{x}_{i}$ to $\boldsymbol{x}_{j}$.

and $\dot{d}_{j}$ which express the velocities of the respective contact points projected into the direction of the collision, see Fig. 3. Positive directions for both $\dot{d}_{i}$ and $\dot{d}_{j}$ are defined from $\boldsymbol{x}_{i}$ to $\boldsymbol{x}_{j}$. Following this, a desired, standard rigid body robot differential equation [32] can be set up:

$$
\begin{aligned}
& \boldsymbol{M}_{d, i, j}(\boldsymbol{q})\left(\begin{array}{c}
\ddot{d}_{i} \\
\ddot{d}_{j}
\end{array}\right)+\boldsymbol{C}_{i, j}(\boldsymbol{q}, \dot{\boldsymbol{q}}) \dot{\boldsymbol{q}}+\boldsymbol{g}_{i, j}(\boldsymbol{q})=\boldsymbol{F}_{d, i, j}, \\
& \boldsymbol{F}_{d, i, j}=-\boldsymbol{D}_{d, i, j}(\boldsymbol{q})\left(\begin{array}{c}
\dot{d}_{i} \\
\dot{d}_{j}
\end{array}\right)-F_{\mathrm{rep}}\left(d_{i, j}\right)\left(\begin{array}{r}
1 \\
-1
\end{array}\right) .
\end{aligned}
$$

Coriolis and centrifugal effects are comprised by $\boldsymbol{C}_{i, j}(\boldsymbol{q}, \dot{\boldsymbol{q}}) \dot{\boldsymbol{q}} \in \mathbb{R}^{2}$ and gravitational effects are represented by $\boldsymbol{g}_{i, j}(\boldsymbol{q}) \in \mathbb{R}^{2}$. The mass matrix $\boldsymbol{M}_{d, i, j}(\boldsymbol{q}) \in \mathbb{R}^{2 \times 2}$ contains the reflected inertias at the contact points in the direction of the collision, i. e.,

$$
\boldsymbol{M}_{d, i, j}(\boldsymbol{q})=\left(\begin{array}{cc}
M_{d, i}(\boldsymbol{q}) & 0 \\
0 & M_{d, j}(\boldsymbol{q})
\end{array}\right) .
$$

The damping matrix $\boldsymbol{D}_{d, i, j}(\boldsymbol{q}) \in \mathbb{R}^{2 \times 2}$ in (10) is designed such that a desired damping behavior in the modal space is realized.

The following derivation transforms the known joint mass matrix of the manipulator into $M_{d, i}(\boldsymbol{q})$. Scalar $M_{d, j}(\boldsymbol{q})$ is obtained analogously. Afterwards, the configuration dependent damping matrix is derived.

1) Mass Projection $M_{d, i}(\boldsymbol{q})$ : We consider the general relation between joint torques $\tau \in \mathbb{R}^{n}$ and joint accelerations $\ddot{q}$.

$$
\boldsymbol{M}(\boldsymbol{q}) \ddot{\boldsymbol{q}}+\boldsymbol{c}(\boldsymbol{q}, \dot{\boldsymbol{q}})=\boldsymbol{\tau},
$$

where $\boldsymbol{M}(\boldsymbol{q}) \in \mathbb{R}^{n \times n}$ is the corresponding joint mass matrix. Coriolis and centrifugal effects are represented by $\boldsymbol{c}(\boldsymbol{q}, \dot{\boldsymbol{q}})$. Additionally, the well-known transformation from joint space to Cartesian space via the Jacobian matrix $\boldsymbol{J}_{x, i}(\boldsymbol{q}) \in \mathbb{R}^{3 \times n}$ with respect to an arbitrary point $\boldsymbol{x}_{i}^{\mathcal{C}(i)}(\boldsymbol{q}) \in \mathbb{R}^{3}$ is required:

$$
\begin{aligned}
\dot{\boldsymbol{x}}_{i}^{\mathcal{C}(i)} & =\boldsymbol{J}_{x, i}(\boldsymbol{q}) \dot{\boldsymbol{q}}, \\
\boldsymbol{\tau} & =\boldsymbol{J}_{x, i}^{T}(\boldsymbol{q}) \boldsymbol{F}_{x, i}^{\mathcal{C}(i)} .
\end{aligned}
$$

The Cartesian velocity is expressed by $\dot{\boldsymbol{x}}_{i}^{\mathcal{C}(i)}$, and $\boldsymbol{F}_{x, i}^{\mathcal{C}(i)} \in \mathbb{R}^{3}$ represents an external force applied at $\boldsymbol{x}_{i}^{\mathcal{C}(i)}$. As our goal is to end up in the direction of the collision $e_{i}$, we define the mapping

$$
\boldsymbol{J}_{d, i}\left(\boldsymbol{e}_{i}\right)=\boldsymbol{e}_{i}^{T}
$$

between motions along $\boldsymbol{e}_{i}$ and the Cartesian coordinates of the contact point. Combining (15) with (13) delivers the complete projection.

$$
\dot{d}_{i}=\boldsymbol{J}_{d, i}\left(\boldsymbol{e}_{i}\right) \dot{\boldsymbol{x}}_{i}^{\mathcal{C}(i)}=\underbrace{\boldsymbol{J}_{d, i}\left(\boldsymbol{e}_{i}\right) \boldsymbol{J}_{x, i}(\boldsymbol{q})}_{\boldsymbol{J}_{i}(\boldsymbol{q})} \dot{\boldsymbol{q}}
$$

with $\boldsymbol{J}_{i}(\boldsymbol{q}) \in \mathbb{R}^{1 \times n}$ expressing the resultant Jacobian row vector which relates the joint space to the distance-space as introduced in (6). Deriving $\dot{d}_{j}$ is done analogously with $e_{j}$ being oriented in the same direction as $\boldsymbol{e}_{i}$.

Based on (16), the acceleration constraint is obtained by differentiation w.r.t. time:

$$
\ddot{d}_{i}=\boldsymbol{J}_{i}(\boldsymbol{q}) \ddot{\boldsymbol{q}}+\dot{\boldsymbol{J}}_{i}(\boldsymbol{q}, \dot{\boldsymbol{q}}) \dot{\boldsymbol{q}}
$$

Combining (12), (14), (16) and (17) leads to

$$
\begin{gathered}
\ddot{d}_{i}=\dot{\boldsymbol{J}}_{i}(\boldsymbol{q}, \dot{\boldsymbol{q}}) \dot{\boldsymbol{q}}-\boldsymbol{J}_{i}(\boldsymbol{q}) \boldsymbol{M}(\boldsymbol{q})^{-1} \boldsymbol{c}(\boldsymbol{q}, \dot{\boldsymbol{q}})+ \\
+\underbrace{\boldsymbol{J}_{i}(\boldsymbol{q}) \boldsymbol{M}(\boldsymbol{q})^{-1} \boldsymbol{J}_{i}^{T}(\boldsymbol{q})}_{M_{d, i}(\boldsymbol{q})^{-1}} F_{x, i}^{d} .
\end{gathered}
$$

Herein $M_{d, i}(\boldsymbol{q})$ expresses the scalar mass of point $\boldsymbol{x}_{i}^{\mathcal{C}(i)}$ to be accelerated in the direction of the collision. Due to the transformation, $F_{x, i}^{d} \in \mathbb{R}$ now describes a scalar force acting at $\boldsymbol{x}_{i}^{\mathcal{C}(i)}$. From a computational point of view, the calculation of $M_{d, i}(\boldsymbol{q})$ is not expensive as the last inversion refers to a scalar. Moreover, inverting the joint mass matrix $\boldsymbol{M}(\boldsymbol{q})$ has to be done only once per sample time, independent of the number of contact point pairs.

2) Damping Matrix $\boldsymbol{D}_{d, i, j}(\boldsymbol{q})$ : Utilizing $\boldsymbol{M}_{d, i, j}(\boldsymbol{q})$ allows to apply desired damping ratios $\zeta_{1}$ and $\zeta_{2}$ in the modal space (decoupled dynamics). However, since (9) is nonlinear, we linearize around the actual working point $d_{i, j}^{*}=f\left(\boldsymbol{q}^{*}\right)$, under the additional assumption of a quasi-static analysis, $\dot{d}_{i}^{*}=\dot{d}_{j}^{*}=$ 0 . That leads to

$$
\begin{aligned}
& \boldsymbol{M}_{d, i, j}\left(\boldsymbol{q}^{*}\right)\left(\begin{array}{c}
\delta \ddot{d}_{i} \\
\delta \ddot{d}_{j}
\end{array}\right)=\boldsymbol{F}_{d, i, j}^{*}, \\
& \boldsymbol{F}_{d, i, j}^{*}=-\boldsymbol{D}_{d, i, j}^{*}\left(\begin{array}{c}
\delta \dot{d}_{i} \\
\delta \dot{d}_{j}
\end{array}\right)-\boldsymbol{K}_{d, i, j}^{*}\left(\begin{array}{c}
\delta d_{i} \\
\delta d_{j}
\end{array}\right),
\end{aligned}
$$

where $\boldsymbol{F}_{d, i, j}^{*}$ denotes the control input which includes the damping and the repulsion. The local stiffness matrix $\boldsymbol{K}_{d, i, j}^{*} \in$ $\mathbb{R}^{2 \times 2}$ is defined as follows:

$$
\begin{aligned}
\boldsymbol{K}_{d, i, j}^{*} & =\left.\left(\begin{array}{r}
1 \\
-1
\end{array}\right) \frac{\partial F_{\mathrm{rep}}\left(d_{i, j}\right)}{\partial d_{i, j}}\right|_{d_{i, j}=d_{i, j}^{*}} \cdot \frac{\partial d_{i, j}}{\partial\left(d_{i} d_{j}\right)} \\
& =\left.\frac{\partial^{2} V_{\mathrm{rep}, i, j}\left(d_{i, j}\right)}{\partial d_{i, j}^{2}}\right|_{d_{i, j}=d_{i, j}^{*}} \cdot\left(\begin{array}{rr}
1 & -1 \\
-1 & 1
\end{array}\right) .
\end{aligned}
$$

Notice that the gravitational effects from (9) are omitted in (19) as we assume a separate static gravity compensation in the overall controller. The local damping behavior is specified by $\boldsymbol{D}_{d, i, j}^{*} \in \mathbb{R}^{2 \times 2}$. Based on (19), (20), and (21), various methods from linear algebra theory can be applied in order to realize the desired damping ratios $^{3}$. We have chosen the Double Diagonalization approach by Albu-Schäffer et al. [33]. The damping matrix can be formally written as

$$
\boldsymbol{D}_{d, i, j}^{*}=\mathcal{D}\left(\boldsymbol{M}_{d, i, j}\left(\boldsymbol{q}^{*}\right), \boldsymbol{K}_{d, i, j}^{*}, \zeta_{1}, \zeta_{2}\right) \text {. }
$$

It will be computed and applied in each control cycle. At this point it shall also be mentioned that it is straightforward

\footnotetext{
${ }^{3}$ Although the damping design is done for the linear system, it is still valid for the analysis of the nonlinear system as the term only appears in the derivative of the Lyapunov function.
} 

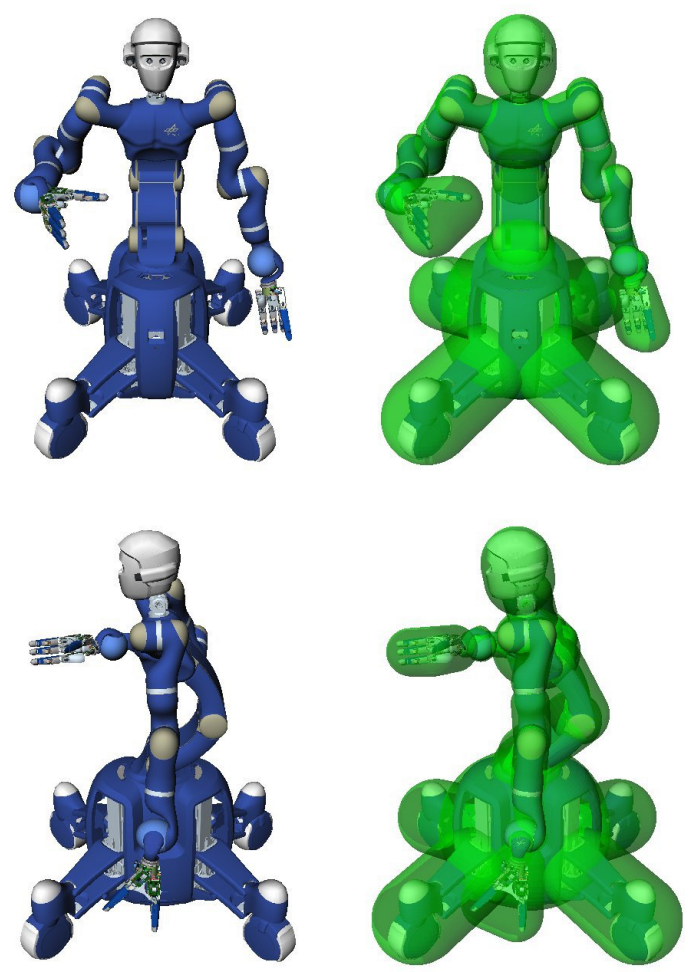

Fig. 4. Geometric collision model of the robot consisting of 28 bounding volumes (left arm: 8 , right arm: 8 , mobile base: 5 , torso: 4 , head: 2 , floor: 1 ). The volumes are spheres, rounded cylinders, and bodies obtained by unrolling spheres on triangles.

to design a relative damping instead of an absolute one by slightly modifying (9) and (10). A benefit of that choice would be a reduction of the dimension of the self-collision avoidance (per contact point pair) from two to one.

\section{Collision Avoidance Torques}

The procedure has to be applied to each of the $n_{\mathrm{p}}$ contact point pairs. The collision avoidance torques due to repulsion and damping effects w.r.t. the actual values of $\boldsymbol{q}, d_{i, j}, \dot{d}_{i}$, and $\dot{d}_{j}$ are

$$
\boldsymbol{\tau}_{\text {coll }}=\sum_{i=1}^{n_{\mathrm{p}}}\left(\begin{array}{c}
\boldsymbol{J}_{i}(\boldsymbol{q}) \\
\boldsymbol{J}_{j}(\boldsymbol{q})
\end{array}\right)^{T}\left(\left(\begin{array}{c}
-F_{\text {rep }}\left(d_{i, j}\right) \\
F_{\text {rep }}\left(d_{i, j}\right)
\end{array}\right)-\boldsymbol{D}_{d, i, j}^{*}\left(\begin{array}{c}
\dot{d}_{i} \\
\dot{d}_{j}
\end{array}\right)\right)
$$

\section{Experimental Validation of the Self-Collision Avoidance}

We applied the approach to the humanoid robot Justin of the German Aerospace Center (DLR). The geometric model for contact point calculations is illustrated in Fig. 4 (right). The respective distance computation algorithm is based on the formulation by Gilbert et al. [31] and has been adapted to the system in [29]. In order to facilitate real-time applicability, the collision model is calculated once per control cycle $(1 \mathrm{~ms})$ incorporating 302 pairs of links. The computing time lies between $0.3 \mathrm{~ms}$ and $0.4 \mathrm{~ms}$ on an Intel Core2Duo Processor T7400 (2.16 GHz).
TABLE I

PARAMETERIZATION FOR THE EXPERIMENT \#1 ON SELF-COLLISION AVOIDANCE

\begin{tabular}{|c|c|c|c|}
\hline$F_{\max }$ & $d_{0}$ & $n_{\mathrm{p}}$ & $\zeta=\zeta_{1}=\zeta_{2}$ \\
\hline \hline $25 \mathrm{~N}$ & $0.15 \mathrm{~m}$ & 35 & $0,0.7,1.0,1.3$ \\
\hline
\end{tabular}
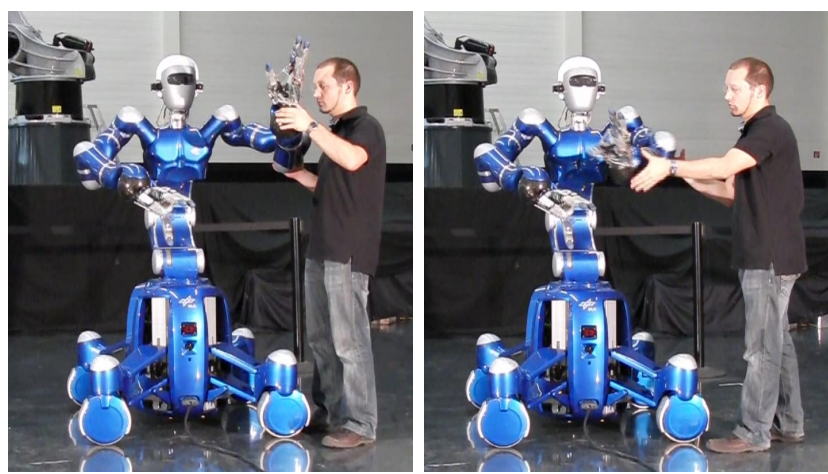

Fig. 5. Initial configuration (left) and snapshot during the experiment \#1 (right) on self-collision avoidance. Except for the left arm (7 DOF), all other joints are locked in order to facilitate repeatability of the experiment.

For experiment \#1, the parameterization in Table I is taken. For each run, a different damping behavior is chosen. Throughout, the robot is controlled in gravity compensation mode. Except for the left arm (7 DOF), all other joints are locked. Fig. 5 (left) shows the initial configuration. The user is required to feed kinetic energy into the system (right), throwing the left forearm onto the right arm. A total number of 14 penetrated potential fields is counted during the measurements including the arms, the torso, the mobile base, and the head. The most critical ones are shown in Fig. 6. They refer to the sets "left hand - right hand" (left column plots) and "left hand right wrist" (right column plots). The implementation for this experiment allows a maximum number of 35 simultaneous potential field penetrations. That number can be varied, but in experiments it revealed to be sufficient such that each penetrated potential field is taken into account, independent of the configuration ${ }^{4}$.

As the user inserted about the same amount of kinetic energy in all of the four scenarios ${ }^{5}$, the penetration of the potential fields is significantly smaller while damping is active. The returning velocities of the links are affected by the choice of $\zeta$ as it can be seen in the upper plots in Fig. 6. Without dissipation by damping forces, no energy is taken out of the system. Due to the potential design, the repulsive forces are continuously differentiable. Since the damping directly depends on the local potential stiffness, the damping forces are continous but not continuously differentiable as it can be observed in the bottom plots in Fig. 6.

Since we assigned pure gravity compensation, the potential

${ }^{4}$ Of course, that is only valid if the potential fields are designed with a proper and reasonable extent as done here, e.g. $d_{0}=0.15 \mathrm{~m}$. As the number of possible pairs in the geometric collision model is $302, d_{0} \rightarrow \infty$ would require 302 instead of 35 pairs to be taken into account.

${ }^{5}$ The energies which are absorbed by the most relevant potential field in all four cases, i.e., "left hand - right hand", have a maximum deviation of $<12 \%$. 

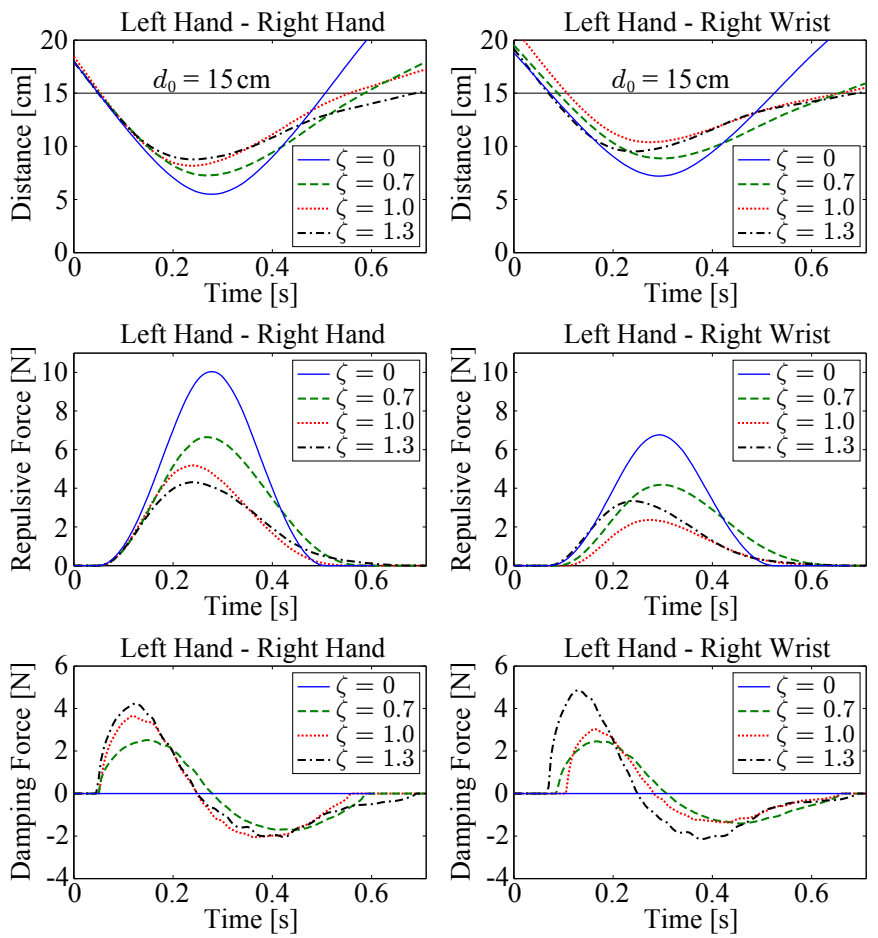

Fig. 6. Experiment \#1 on self-collision avoidance: Repulsion between left hand and right hand/wrist for an undamped $(\zeta=0)$, underdamped $(\zeta=0.7)$, critically damped $(\zeta=1.0)$, and overdamped $(\zeta=1.3)$ system. These two combinations are a selection of a total number of 14 active contact point pairs.

field $V_{\text {rep,tot }}(\boldsymbol{q})$ does not compete with any other objectives. Taking account of additional tasks and constraints raises the question of a proper priority order or task hierarchy so as to avoid undesired and undefined competitions.

The interested reader may refer to the supporting video for more complex self-collision avoidance scenarios with a larger number of actuated DOF.

\section{REALIZING A TASK HIERARCHY WITH UNILATERAL CONSTRAINTS}

This section provides a redundancy resolution to integrate the unilateral self-collision avoidance into an arbitrary task hierarchy. It is fundamentally based on null space projection techniques by Khatib [12], and Siciliano and Slotine [14] to handle the numerousness of DOF. The major extension is the consideration of online activation/deactivation processes of unilateral constraints.

First, we recall some fundamentals about null space projections and state the problem of discontinuities when applying classical methods to unilateral constraints. Afterwards, we derive our solution based on [30].

\section{A. Fundamentals and Problem Statement}

A common way to induce a task hierarchy is to utilize null space projection techniques [12], [14]. A high priority task with dimension $m$ is described by a virtual constraint $\boldsymbol{f}(\boldsymbol{q})=$ 0, to which a Jacobian matrix $\boldsymbol{J}=\frac{\partial \boldsymbol{f}(\boldsymbol{q})}{\partial \boldsymbol{q}} \in \mathbb{R}^{m \times n}$ belongs. Initially, $\boldsymbol{J}$ is supposed to be non-singular. In the redundant case, i. e. $m<n$, a torque from lower priority tasks can be projected into the null space of $\boldsymbol{J}$ via the projection matrix

$$
\boldsymbol{N}=\boldsymbol{I}-\boldsymbol{J}^{T} \boldsymbol{J}^{+T}
$$

where $\boldsymbol{J}^{+}$denotes the Moore-Penrose pseudoinverse of $\boldsymbol{J}$. Note that this is only one specific choice for the definition of the null space ${ }^{6}$. From a numerical perspective, the inversion is mostly done by utilizing a singular value decomposition (SVD):

$$
\boldsymbol{J}=\boldsymbol{U} \boldsymbol{S} \boldsymbol{V}^{T}
$$

with $\boldsymbol{U} \in \mathbb{R}^{m \times m}$ being a unitary matrix, $\boldsymbol{S} \in \mathbb{R}^{m \times n}$ a rectangular diagonal matrix containing the singular values $\sigma_{1}$ to $\sigma_{m}$, and $\boldsymbol{V} \in \mathbb{R}^{n \times n}$ a unitary matrix. Following this, the well-known representation of $\boldsymbol{J}^{+}$based on SVD components is

$$
\boldsymbol{J}^{+}=\boldsymbol{V} \boldsymbol{S}^{+} \boldsymbol{U}^{T} .
$$

Inverting $S$ in (26) is commonly realized by inverting the diagonal elements and cancelling the singular values less than a specified tolerance $\varepsilon$. At this point, the occurrence of discontinuities becomes evident. If the rank of the Jacobian matrix changes, threshold $\varepsilon$ of one or more singular values is crossed. That effect propagates back to (24) and causes a discontinuous control law on torque level.

It shall be noted that cancelling the singular values less than $\varepsilon$ is an arbitrary choice to deal with the singularity while inverting the diagonal elements of $\boldsymbol{S}$. Another possibility to handle that problem is to set a lower bound for the singular values before inverting them. Moreover, an established method from the field of inverse kinematics is to utilize damped leastsquares techniques [23], i. e.,

$$
\boldsymbol{J}^{\dagger}=\boldsymbol{J}^{T}\left(\boldsymbol{J} \boldsymbol{J}^{T}+\lambda \boldsymbol{I}\right)^{-1} .
$$

Herein, ${ }^{\dagger}$ denotes the damped inversion operator. The damping parameter $\lambda \in \mathbb{R}^{+}$is specified to smooth the transition. So far, various different approaches concerning damped leastsquares methods have been proposed. First solutions suggested a constant damping factor [24] but quickly revealed a crucial problem: Accuracy of the inverse away from the singularity and ensuring a smooth transition simultaneously is fairly unfeasible. Later designs based upon variable damping factors, e. g., dependent on the distance to the singularity [25] or its time derivative [26]. However, several problems remain. Beside the fact that $\boldsymbol{J}^{\dagger}$ is not a correct inverse of $\boldsymbol{J} \forall \lambda \neq 0$, the choice of the damping parameter is not intuitive and the direct consequence on physical values of the system is not clear.

\section{B. Continuous Null Space Projection Shaping}

When transitions occur between active and inactive selfcollision potential fields, the respective Jacobian matrix changes rank and leads to a discontinuous control law if that issue is not handled appropriately. In the following, we will systematically shape the transition behavior in order to limit

\footnotetext{
${ }^{6}$ Another frequently used inversion in robotics is called dynamically consistent [12] which is obtained by taking $\boldsymbol{M}(\boldsymbol{q})$ into account.
} 
the torque derivative during the transition phase. That closes the gap between the mathematical mechanisms of the projector calculation and the physical values of the real system. We start with a 1 DOF system and extend the method step by step, right up to the general case. Our approach does not provide a dynamically consistent [12] but a static [34] null space projection. However, as shown in [34], the static null space projection can be augmented to a dynamically consistent one by incorporating the inertia distribution.

An intuitive interpretation of the null space projector can be performed by considering the SVD component notation of (24):

$$
\begin{aligned}
\boldsymbol{N} & =\boldsymbol{I}-\boldsymbol{V} \boldsymbol{S}^{T} \boldsymbol{U}^{T}\left(\boldsymbol{V} \boldsymbol{S}^{+} \boldsymbol{U}^{T}\right)^{T} \\
& =\boldsymbol{I}-\boldsymbol{V} \underbrace{\boldsymbol{S}^{T} \boldsymbol{S}^{+T}}_{\boldsymbol{A}} \boldsymbol{V}^{T}
\end{aligned}
$$

Herein

$$
\boldsymbol{A}=\operatorname{diag}\left(a_{1}, a_{2}, \ldots, a_{m}, \mathbf{0}_{1 \times(n-m)}\right)
$$

with

$$
a_{i}=\left\{\begin{array}{ll}
0 & \text { if } \sigma_{i}<\varepsilon \\
1 & \text { otherwise }
\end{array} \quad \forall 1 \leq i \leq m .\right.
$$

It becomes evident in (29) that only the right-singular vectors comprised by $\boldsymbol{V}$ are relevant for the null space projector $^{7}$, whereas the left-singular vectors in $\boldsymbol{U}$ and the absolute singular values in $\boldsymbol{S}$ do not have any influence on the result. The latter can be shown when considering the socalled activation matrix $\boldsymbol{A} \in \mathbb{R}^{n \times n}$. That matrix is supposed to contain either 1 (active) or 0 (inactive) diagonal elements. The $i$-th diagonal element refers to the $i$-th column vector in $\boldsymbol{V}$ and either activates that direction or locks it.

Since the absolute singular values of the Jacobian matrix are not of interest, we just require the directions of the constraint. In the following, the notation $\boldsymbol{J}_{m \times n} \in \mathbb{R}^{m \times n}$ for the Jacobian matrix will be used.

\section{Considering a 1 DOF System}

A $n=1$ DOF system is illustrated in Fig. 7. The depicted mass may move horizontally on the chain dotted line, the location is described by $z$. At $z=z_{\text {uni }}$, a repulsive potential field is penetrated whose purpose it is to avoid a collision with the wall. The potential shall be the high priority task, whereas arbitrary tasks define the mass behavior in the null space of the collision avoidance task.

We provide the Jacobian matrix of the primary task

$$
\boldsymbol{J}=\sigma \boldsymbol{J}_{1 \times 1}
$$

where the direction $\boldsymbol{J}_{1 \times 1}=[1]$ is invariant and the singular value $\sigma$ is extracted from $\boldsymbol{J}$ beforehand. A SVD of (32) leads to (25) with $\boldsymbol{U}=[1], \boldsymbol{S}=[\sigma], \boldsymbol{V}=[1]$. Applying (29) delivers

$$
N=1-S S^{+}=1-A
$$

with all matrices degenerated to scalars.

\footnotetext{
${ }^{7}$ Actually, only the first $m$ column vectors in $\boldsymbol{V}$ are relevant here. Thus, a reduced SVD suffices to compute $\boldsymbol{V}$.
}

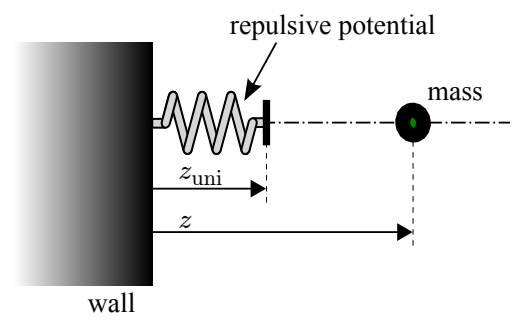

Fig. 7. 1 DOF system with unilateral constraint (repulsive potential) to avoid a collision of the mass with the wall.

\section{Desired Transition Behavior for the 1 DOF system}

The discontinuity stated in (31) raises the question: Which behavior for the null space projector do we actually desire? Evidently, a continuous transition between 0 and 1 is required at least. Moreover, a desired behavior is to specifically influence the values which are affected in the end: the projected torques or their derivatives, respectively. In this context we shape the projector (33) such that $N=N_{\text {des }}(z)$. The variable $z$ determines the state of the activation of the unilateral constraint. An example for $z$ is the distance between an object and a potential collision partner to describe the closeness to a collision, see Fig. 7.

Following (33), the required and desired activator is defined by

$$
a_{1, \mathrm{des}}=1-N_{\mathrm{des}}(z)
$$

A desired behavior could be to limit $\partial N_{\text {des }}(z) / \partial t$. Projecting a secondary task $\tau_{\text {sec }}$ into the null space of the primary task (hierarchy level 1), we obtain the control input

$$
\begin{aligned}
& \tau_{\text {sec,proj }}=N_{\text {des }}(z) \tau_{\text {sec }} \\
& \dot{\tau}_{\text {sec,proj }}=\frac{\partial N_{\mathrm{des}}(z)}{\partial z} \frac{\partial z}{\partial t} \tau_{\text {sec }}+N_{\text {des }}(z) \dot{\tau}_{\text {sec }}
\end{aligned}
$$

Obviously, $N_{\text {des }}(z)$ must be at least of type $C^{1}$ in order to ensure continuity of (36). For the further analysis, we make the following assumptions:

1) We neglect $\dot{\tau}_{\text {sec }}$. This is valid as we assume the transition to be faster than the changing of torque $\tau_{\text {sec }}$ from a regular task.

2) A maximum or worst case $\tau_{\text {sec }}$ can be specified. If this is not possible, an online calculation or measurement is provided.

3) We are able to estimate a maximum or worst case value for $\partial z / \partial t$. If this is not possible, we are able to calculate or measure $\partial z / \partial t$ online.

We suggest a piecewise defined function for $N_{\mathrm{des}}(z)$ containing a third order polynomial, i.e.,

$$
\begin{gathered}
N_{\text {des }}(z)= \begin{cases}0 & \text { if } z<z_{1} \\
g(z) & \text { if } z_{1} \leq z \leq z_{2} \\
1 & \text { otherwise }\end{cases} \\
g(z)=k_{1} z^{3}+k_{2} z^{2}+k_{3} z+k_{4}
\end{gathered}
$$

with $\left[z_{1}, z_{2}\right]$ defining the interval from full locking to unconstrained null space projection. Limiting $N_{\max }^{\prime}=\max \left(\partial N_{\operatorname{des}}(z) / \partial z\right)$ allows to "stretch" the 
TABLE II

CONSTRAINTS FOR THE TRANSITION FUNCTION

\begin{tabular}{|c|c|c|c|}
\hline & $z_{1}$ & $z_{2}$ & $\left(z_{1}+z_{2}\right) / 2$ \\
\hline$g(z)$ & 0 & 1 & \\
\hline$g^{\prime}(z)$ & 0 & 0 & $N_{\max }^{\prime}$ \\
\hline
\end{tabular}
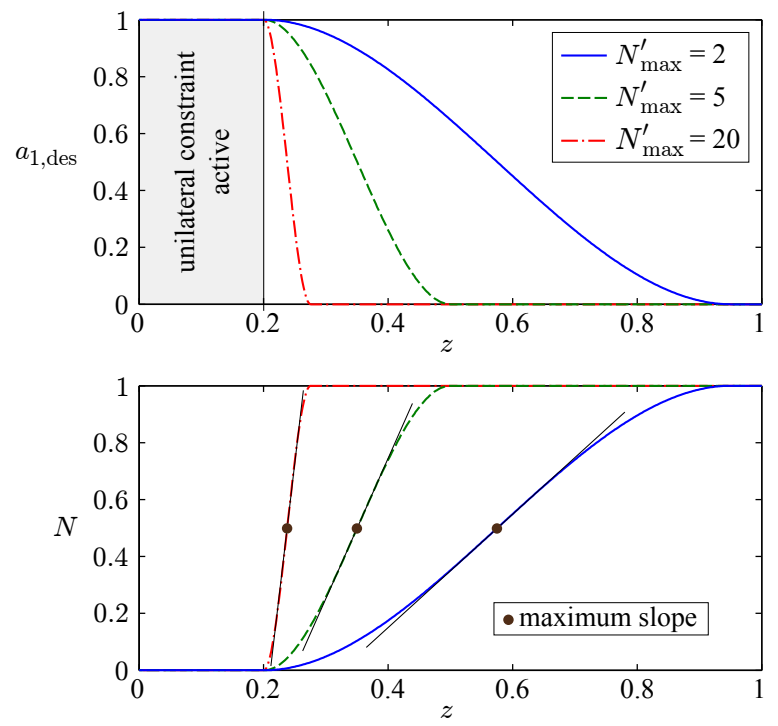

Fig. 8. Examples of transition shaping in case of a 1 DOF system as depicted in Fig. 7. The plots illustrate the compromise between smoothness of the transition and the interval size.

torque change/discontinuity over a well-defined range. More precisely, a maximum torque derivative $\dot{\tau}_{\text {sec,proj,max }}$ can be specified $^{8}$ :

$$
N_{\max }^{\prime}=\dot{\tau}_{\text {sec,proj,max }}\left|\frac{\partial z}{\partial t} \tau_{\text {sec }}\right|^{-1} .
$$

One choice for $\dot{\tau}_{\text {sec,proj,max }}$ is a parameterization according to the performance of the torque control loop. We set the conditions in Table II for (38). That over-determined system of equations can be solved by adding the range $\left\{z_{2}-z_{1}\right\}$ to the set of unknown parameters $\left\{k_{1}, k_{2}, k_{3}, k_{4}\right\}$ in (38).

Notice that limiting $N_{\max }^{\prime}$ for (38) is a conservative approach to limit $\dot{\tau}_{\text {sec,proj }}$ as the maximum slope of $N$ is only reached once within the transition interval. It will be shown in the following simulation that using a third order polynomial is only marginally more conservative than the fastest continuous transition, i.e., an affine function, but it has the advantage of a significantly smoother behavior.

Fig. 8 shows results for $N_{\max }^{\prime}=2,5,20$. The upper plots depict the activator. At $z=z_{1}=0.2$, the unilateral constraint is fully activated and the DOF is locked for all lower priority tasks than the highest one. The parameterization of $N_{\max }^{\prime}$ can be identified in the bottom plots when regarding the maximum slope of $N$.

As described above, other transition functions than (38) may be used. Applying an affine relation would bring the benefit of a constant $\partial N(z) / \partial z$ within the transition phase instead of

\footnotetext{
${ }^{8}$ If $\partial z / \partial t$ and $\tau_{\text {sec }}$ are taken from measurements or estimations online, a loop is closed. In order to ensure passivity a separate proof has to be made.
}

the quadratic ones shown in Fig. 8 (bottom). It can be shown that the interval size would reduce to $2 / 3$. However, a lack of smoothness would result at the beginning of the interval and at $z=0.2$. Notice also that $z_{1}$ does not have to be set equal to $z_{\text {uni }}$ from Fig. 7 .

In our implementations, we define $z_{1}$ as the point of full activation of the primary task. That is a design choice of the hierarchy concept. By defining $N_{\max }^{\prime}$, the location of $z_{2}$ becomes determined uniquely.

\section{E. Considering $a(1 \times n)$ Constraint}

The 1 DOF case is trivial as the null space projector equals a complete fade-out of the secondary task torque at the activation point of the constraint. Now, we will extend the method to the nontrivial $(1 \times n)$ case such that $\boldsymbol{J}=\sigma \boldsymbol{J}_{1 \times n} \in \mathbb{R}^{1 \times n}$. Hence, $\boldsymbol{J}_{1 \times n}$ is already normalized. Starting from (29), we apply the desired diagonal activation matrix $\boldsymbol{A}_{\mathrm{des}}$ and obtain

$$
\begin{aligned}
\boldsymbol{N} & =\boldsymbol{I}-\boldsymbol{V} \boldsymbol{A}_{\mathrm{des}} \boldsymbol{V}^{T} \\
& =\boldsymbol{I}-\boldsymbol{J}_{1 \times n}^{T} a_{1, \mathrm{des}} \boldsymbol{J}_{1 \times n} .
\end{aligned}
$$

Only the first element $a_{1 \text {,des }}$ of $\boldsymbol{A}_{\mathrm{des}}$ is important. As $\boldsymbol{J}$ is a row vector here, $\boldsymbol{v}_{1}=\boldsymbol{J}_{1 \times n}^{T}$. That turns the method into a very computationally efficient technique.

In various redundancy resolutions variable weights or activators are used in terms of null space computations [35], [16], [19], [17]. The purposes and conditions of the activation strongly differ from each other. In [35], for example, a weighted least-norm solution to avoid joint limits is proposed. It bases on a configuration dependent weighting matrix in order to scale between the different joint contributions. In that approach, the joint limit avoidance is applied on the lowest level where discontuinities never occur. A time-based parameterization for blending and fading out of tasks is proposed in [16]. As we have clarified in the preceding paragraphs, we are in contrast to existing techniques since we provide direct control over the critical directions via $\boldsymbol{A}_{\text {des }}$ and are able to design the transition behavior according to physical limitations of the actuators.

\section{F. Desired Transition Behavior in the $(1 \times n)$ and $(m \times n)$ Case}

One approach to handle the complexity in the $(m \times n)$ case is to decompose the lower level torques $\tau_{\mathrm{sec}}$ by projecting them into the critical directions of $\boldsymbol{V}$. The contributions in these critical directions can be used as a basis for the methods from Sec. III-D, see assumption 2). Notice that an online decomposition and a feedback into the generation process of $\boldsymbol{A}_{\mathrm{des}}$ closes an additional loop. However, as stated in assumption 1) in Sec. III-D, the transition is supposed to be significantly faster than the changing of torques from the lower levels. Thus, the effect is expected to be rather limited. An offline consideration is more conservative but does not close a further loop. At this point it shall be noted that the design in the $(1 \times n)$ case is straightforward when applying such an online decomposition as the critical direction is $\boldsymbol{J}_{1 \times n}$. 


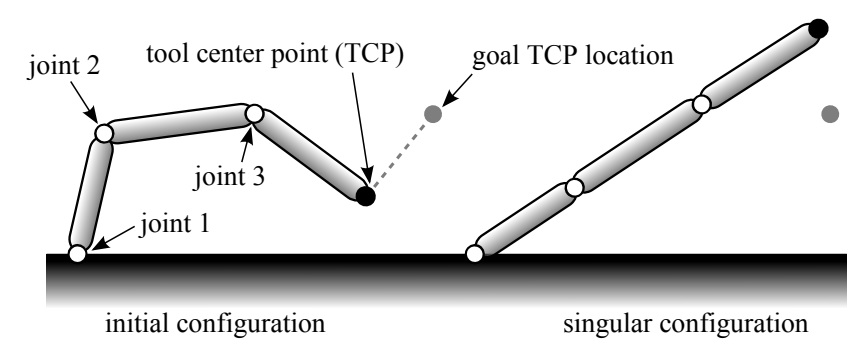

Fig. 9. Schematic representation of the planar 3 DOF system used for the simulations.
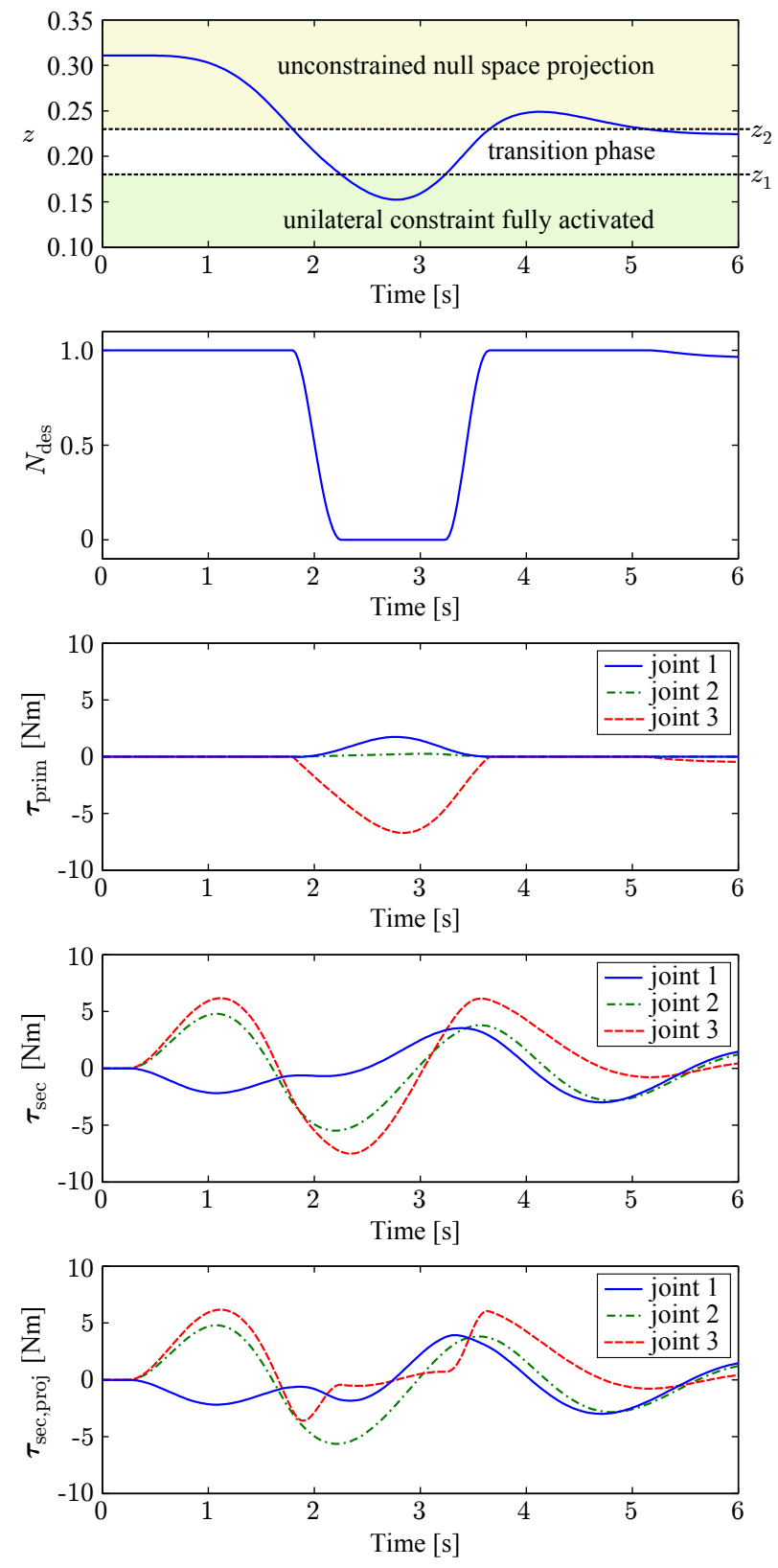

Fig. 10. Activation of a unilateral constraint with $N_{\max }^{\prime}=30$ in case of a 3 DOF system simulation. The system is designed with low damping to provoke several penetrations of the transition area. The state of activation of the primary task is given by $z=m_{\mathrm{kin}}(\boldsymbol{q})$, and the threshold is $z_{2}=m_{0}$.

\section{G. Validation of the Redundancy Resolution in Simulation}

We performed simulations on a planar system as depicted in Fig. 9 (left). It consists of three links and three revolute joints. Viscous joint friction is modeled and the masses are decoupled. As task with low priority, a Cartesian impedance is chosen whose goal it is to lead the tool center point (TCP) to the goal configuration. The respective Jacobian matrix is $\boldsymbol{J}_{\text {sec }}(\boldsymbol{q})$. The primary task is defined by a singularity avoidance which is designed via a repulsive potential field $V_{\text {sing }}(\boldsymbol{q})$ based on the kinematic manipulability measure $m_{\text {kin }}(\boldsymbol{q})$ of the secondary task [36]:

$$
\begin{aligned}
& V_{\mathrm{sing}}(\boldsymbol{q})=\left\{\begin{array}{ll}
k_{s}\left(m_{\mathrm{kin}}(\boldsymbol{q})-m_{0}\right)^{2} & \text { if } m_{\mathrm{kin}}(\boldsymbol{q}) \leq m_{0} \\
0 & \text { if } m_{\mathrm{kin}}(\boldsymbol{q})>m_{0}
\end{array},\right. \\
& m_{\mathrm{kin}}(\boldsymbol{q})=\sqrt{\operatorname{det}\left(\boldsymbol{J}_{\mathrm{sec}}(\boldsymbol{q}) \boldsymbol{J}_{\mathrm{sec}}(\boldsymbol{q})^{T}\right)}
\end{aligned}
$$

The positive scalar factor $k_{s}$ controls the gain of the singularity avoidance. That avoidance with torque command $\boldsymbol{\tau}_{\text {prim }}=-\left(\partial V_{\text {sing }}(\boldsymbol{q}) / \partial \boldsymbol{q}\right)^{T}$ is a unilateral constraint which gets activated if the manipulability measure falls below a specified value $m_{0}$. The primary task Jacobian matrix or the direction, respectively, directly derives from $\tau_{\text {prim. }}$. That simulation example has been chosen to demonstrate the wide applicability of our continuous null space projection concept. Nevertheless, the primary task is basically comparable to a repulsive potential for self-collision avoidance. The Cartesian reference trajectory of the TCP (Fig. 9 left) is designed such that the singularity indicated in Fig. 9 (right) is approached. A conflict between the tasks is provoked. Recall that the singular configuration could never be reached by the Cartesian impedance in a steady state. The primary task would outplay the Cartesian impedance finally. Fig. 10 depicts the results for the first $6 \mathrm{~s}$ of the simulation. In this time interval, a full transition occurs that will be analyzed in the following.

Starting from the initial configuration (Fig. 9 left), the end effector moves towards the singular configuration (right). The primary task gets activated at $t=1.8 \mathrm{~s}$ for the first time. The transition with $N_{\max }^{\prime}=30$ can be observed in the upper plot of Fig. 10. The second plot shows $N_{\text {des }}(t)$. In this experiment, we designed the primary task (third diagram) to start from $z_{2}=m_{0}$ on. The bottom diagrams show the Cartesian impedance torques $\tau_{\text {sec }}$ and their projections $\tau_{\text {sec,proj }}$ into the null space of the primary task. We want to draw attention to the projected torques when the primary task becomes activated. As the singularity avoidance mainly requires intervention at the third joint $(1.8 \mathrm{~s}<t<3.6 \mathrm{~s})$, the respective projected impedance torque alters the most (red, dashed in bottom diagram).

When the unilateral constraint is fully activated, no torque from the Cartesian impedance may remain in primary task direction. According to this, we multiply the projected torques

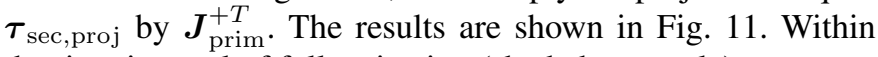
the time interval of full activation (shaded rectangle) no torque comes through. Hence, the condition of an undisturbed priority order is met. The control input $\tau_{\text {cmd }}=\tau_{\text {prim }}+\tau_{\text {sec,proj }}$ is depicted in Fig. 12 (top). For comparison, a discontinuous null space projection based on a classical matrix inversion 


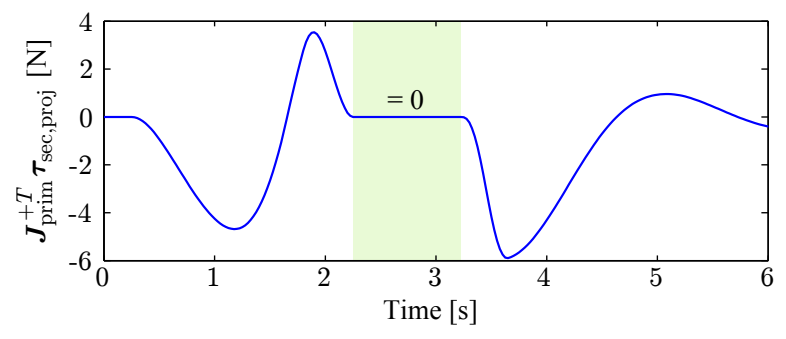

Fig. 11. Projection of the lower priority task torques into the constraint space (continuous null space projection) in case of the 3 DOF system simulation.
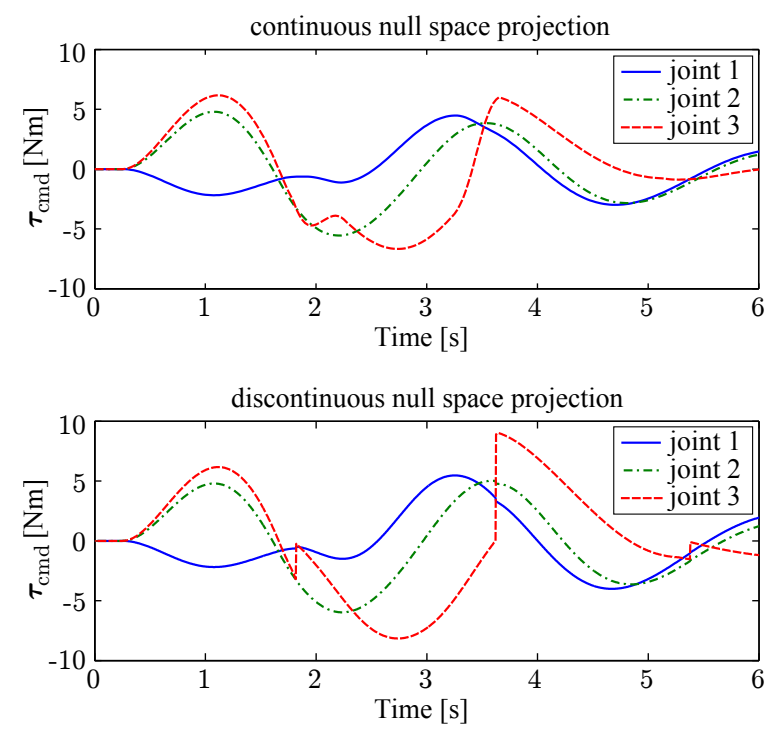

Fig. 12. Control inputs for different null space projection methods in case of the 3 DOF system simulation. The torque discontinuities in the bottom diagram exemplify the problems of common null space projections.

is depicted in the bottom diagram. Significant discontinuities can be observed at $t=1.8 \mathrm{~s}, t=3.6 \mathrm{~s}$ and $t=5.4 \mathrm{~s}$. Applying such commands to a real robotic system would result in unstable behavior as we demonstrated in the experiments in [30].

In this simulation, a steady state in the continuous case is reached after $6 \mathrm{~s}$ asymptotically. In that final configuration, $z$ is a little lower than $z_{2}$ as indicated in Fig. 10 (upper plot). No further full transition occurs after $6 \mathrm{~s}$ since the intervention at $1.8 \mathrm{~s}<t<3.6 \mathrm{~s}$ induced a null space or internal motion that reconfigured the manipulator to comply with the singularity avoidance.

\section{IMPLEMENTATION OF THE SELF-COLlision AVOIDANCE WITHIN A TASK HIERARCHY}

The aim of this section is to merge the presented selfcollision avoidance algorithm with the proposed redundancy resolution. We will provide a method for the task hierarchy design, namely the successive projection method which is a common resolution in whole-body controllers [37]. A detailed comparison to other techniques as well as a stability analysis for the case of velocity controlled systems is done in [38].

The null space of one specific self-collision avoidance direction from (23) described by contact point pair $(i, j)$ can be summarized to

$$
\boldsymbol{N}_{i, j}=\boldsymbol{I}-\boldsymbol{V}_{i, j} \boldsymbol{A}_{i, j, \mathrm{des}} \boldsymbol{V}_{i, j}^{T}
$$

with the activator

$$
\boldsymbol{A}_{i, j, \mathrm{des}}=\left(\begin{array}{ccc}
a_{1, \mathrm{des}}\left(d_{i, j}\right) & 0 & \mathbf{0} \\
0 & a_{1, \mathrm{des}}\left(d_{i, j}\right) & \mathbf{0} \\
\mathbf{0} & \mathbf{0} & \mathbf{0}
\end{array}\right)
$$

of size $(n \times n)$ and

$$
\left(\begin{array}{l}
\boldsymbol{J}_{i}(\boldsymbol{q}) \\
\boldsymbol{J}_{j}(\boldsymbol{q})
\end{array}\right) \boldsymbol{V}_{i, j}=\left(\begin{array}{ccc}
\times & \times & \mathbf{0} \\
\times & \times & \mathbf{0}
\end{array}\right) .
$$

Eq. (44) states that the two Jacobian row vectors are linear combinations of the first two column vectors in $\boldsymbol{V}_{i, j}$. Utilizing (42) offers a multitude of different specifications of the task hierarchy. We formulate the following control law as the general case.

$$
\boldsymbol{\tau}_{\mathrm{cmd}}=\boldsymbol{\tau}_{\mathrm{g}}+\boldsymbol{\tau}_{\mathrm{a}}+\boldsymbol{N}_{\mathrm{a}}\left(\boldsymbol{\tau}_{\text {coll }}+\left(\prod_{i=1}^{n_{\mathrm{p}}} \boldsymbol{N}_{i, h(i)}\right) \boldsymbol{\tau}_{\mathrm{b}}\right) .
$$

In that law, $\tau_{\text {cmd }}$ represents the torque to be commanded to the joints, and $\tau_{\mathrm{g}}$ is the gravity compensation. The subscript a describes a hierarchy level which is placed above the selfcollision avoidance. On the contrary, subscript $\mathrm{b}$ represents a lower priority level. These two (unspecified) levels may consist of their own hierarchical structures. Moreover, the projection into the null space of the structure a, described by $\boldsymbol{N}_{\text {a }}$, may be defined alternatively as for example by the techniques in [14].

\section{DisCUSSION - COMPARISON WITH OTHER APPROACHES AND LiMitaTions OF THE METHOD}

This section addresses the advantages and drawbacks of our method compared to state-of-the-art approaches. That comprises the two subparts, i.e. the self-collision avoidance and the continuous null space projection.

\section{A. Self-Collision Avoidance}

Up to now, implementations of self-collision avoidance on complex humanoid robots are quite sparse. Probably the most relevant approaches are the realizations on HRP-2 [7] and on ASIMO [11]. Both methods as well as our approach ground on the same basic concept of repulsive potentials [6]. However, some fundamental differences exist: Both [7] and [11] operate in the kinematic domain, velocity commands are generated by the controllers. Our algorithm utilizes a force/torque interface. Moreover, damping is not considered there.

A few limitations and restrictions of our concept are mentioned in the following. First, the geometric resolution of the contact point pairs decisively depends on the design of the collision model (Fig. 4). Therefore, the more critical and relevant body parts have been modeled via smaller bounding volumes. That can be seen, for example, when comparing the tightly modeled arms with the roughly modeled mobile base. In a large number of experiments and public demonstrations, the geometric model of Fig. 4 turned out to be a very practicable solution. However, it shall be noted that the design of that 
model is not trivial, and, up to now, it is manually performed. Another problem (theoretically) exists concerning the collision model: As the distance calculation is computationally very expensive, the bounding volumes are kept as simple as possible. In order to avoid discontinuities, they are convex. However, parallel bounding volumes could occur during operation and the respective contact point pair may move (infinitely) fast on the surfaces. Using strictly convex bounding volumes would solve that issue [39]. From a practical point of view, we can say that we have not encountered any problems with the actual model so far. A last issue concerns the torque command of the overall repulsive potential field. As (23) is a superposition of multiple repulsions, the desired behavior, esp. the stiffness specification, is not precisely realized in any case. However, that leads to a more natural and reasonable behavior of the robot. In other words, if several collisions are close, the robot "feels" stiffer which is necessary to avoid the self-collisions. That aspect highlights the need of a proper damping design to increase the robustness once more.

\section{B. Continuous Null Space Projection}

As indicated in Sec. IV, we use the successive projection in our approach. That method is well-established in many multiobjective controllers as the one by Sentis and Khatib [37] or by Lee et al. [17], and it revealed to be very efficient. However, it is a known fact that a successive projection into the null spaces of all higher priority tasks does not lead to a strict compliance with the priority order because the projectors are not compulsorily orthogonal. Thus, the overall successive projection matrix $\boldsymbol{N}_{\text {suc }}$ usually has the property $\boldsymbol{N}_{\text {suc }} \neq \boldsymbol{N}_{\text {suc }} \boldsymbol{N}_{\text {suc }}$ which is a well-known fact. An alternative is the so-called augmented projection [14] which is associated with more expensive numerical computations but it enforces an orthogonality of all involved tasks so that the projection matrix $\boldsymbol{N}_{\text {aug }}$ always fulfills $\boldsymbol{N}_{\text {aug }}=\boldsymbol{N}_{\text {aug }} \boldsymbol{N}_{\text {aug }}$. Especially when a large number of priority levels is defined, the numerical cost increases severely. The sophisticated framework by Mansard et al. [19] is an example of a hierarchy that strictly complies with the priority order while avoiding discontinuities. A detailed discussion and comparison of the successive and the augmented projection is given in [38].

Compared to scaling and blending techniques as [15], wherein secondary tasks may be completely disabled when higher priority constraints become active, we provide an invariance of the remaining directions. In other words, only the contributions in the critical directions are influenced. That also becomes evident through the well-known property

$$
\boldsymbol{v}_{i}^{T} \boldsymbol{N}= \begin{cases}\left(1-a_{i, \mathrm{des}}\right) \boldsymbol{v}_{i}^{T} & \text { if } 1 \leq i \leq m \\ \boldsymbol{v}_{i}^{T} & \text { if } m<i \leq n\end{cases}
$$

which is based on (28). This invariance property of the remaining $n-m$ directions is shown in (46) (bottom line) if a projected task is decomposed into the contributions in the different directions. Moreover, all successive projectionbased approaches as [17], [37] or the method proposed here have the advantage that algorithmic singularities do not appear due to conflicting tasks on different priority levels [38].
TABLE III

PARAMETERIZATION FOR THE EXPERIMENTS ON SELF-COLLISION AVOIDANCE WITHIN THE TASK HIERARCHY

\begin{tabular}{|l||c|c|}
\hline Experiment & $\# 2$ & $\# 3$ \\
\hline \hline$F_{\max }[\mathrm{N}]$ & 30 & 30 \\
\hline$\zeta$ & 0.5 & 0.5 \\
\hline$d_{0}[\mathrm{~m}]$ & 0.10 & 0.10 \\
\hline$z_{1}[\mathrm{~m}]$ & 0.05 & 0.02 \\
\hline$z_{2}[\mathrm{~m}]$ & 0.13 & 0.18 \\
\hline$N_{\max }^{\prime}[1 / \mathrm{m}]$ & 18.8 & 9.4 \\
\hline$K_{\mathrm{t}}[\mathrm{N} / \mathrm{m}]$ & 500 & 500 \\
\hline$K_{\mathrm{r}}[\mathrm{Nm} / \mathrm{rad}]$ & 100 & 100 \\
\hline
\end{tabular}

All augmented projection-based concepts have to deal with that problem additionally. We also want to mention that our approach is real-time applicable. That is not only due to the small numerical cost but also to the easy worst case estimation of the computing time. In contrast to iterative methods as [28] which have been developed for 3D characters mainly, our approach fulfills the condition of real-time applicability. A salient advantage of our concept is the intuitive parameterization of the transition. As stated in Sec. III, we provide the capability of specifying the transient behavior in terms of limitations of the real, physical system. Adapting to the force/torque loops allows to exploit the full performance of the hardware. We provide direct control over the critical directions such that the design is not performed on an abstract level but it is intuitive and easily interpretable. That kind of intuitive parameterization is partly missing in other works [19], [17], [21]. Another major benefit of our approach is its computational efficiency. That characteristic is directly linked to the drawbacks caused by the successive projection method as stated above. Since we use the simple form of (42), any computational methods, e.g. the SVD, can be reduced to a minimum ${ }^{9}$ at the cost of the strictness of the hierarchy.

The kinematic approach by Lee et al. [17] uses a blending on the control input level (task velocities) instead of modifying the inversion. Although the strategy is fundamentally different, the approach could be adapted to the dynamical case and applied to a hierarchy as used in this work.

\section{EXPERIMENTS ON THE HUMANOID JUSTIN}

To validate our approach, we implemented the algorithms on the humanoid Justin. The robot has 51 actuated degrees of freedom and torque sensors in most of the upper body joints ${ }^{10}$. That allows to access a joint torque interface. In the following experiments, only the right arm (7 DOF) is active, all other joints are locked, and the priority order is set as follows:

- High priority: Self-collision avoidance $\tau_{\text {coll }}$ for the whole upper body of the manipulator and gravity compensation.

- Low priority: 6 DOF Cartesian impedance applied to the right TCP.

\footnotetext{
${ }^{9}$ As stated in [27], a trimmed-down or reduced SVD is sufficient to compute the projector.

${ }^{10}$ Only the two neck joints are not equipped with torque sensors.
} 
1) Experiment \#2: The parameterization is given in Table III. The parameters $K_{\mathrm{t}}$ (translational) and $K_{\mathrm{r}}$ (rotational) define the Cartesian stiffnesses which are applied in the three translational and rotational directions. The Cartesian impedance is projected into the null space of the self-collision avoidance between the left and the right hand. That contact point pair is the most critical one here. Thus, $z$ is chosen to be the distance $d_{i, j}$ between these links. The brakes of the left arm are engaged. The initial configuration of the robot is shown in Fig. 15 (a). The snapshots depict the motion of the robot up to the goal location of the right TCP, see Fig. 15 (e). Obviously, the right hand is repelled from the left one during the motion. After reaching the goal location, the right TCP is assigned to move to the initial location again.

The collision avoidance commands during the motion can be observed in Fig. 13 (top left). Below, the distance between left and right hand is plotted. Since the avoidance "disturbs" and filters the Cartesian impedance, a deviation between commanded and real TCP location results. The respective translational error is also depicted in this diagram. A higher Cartesian stiffness would reduce the translational error but at the point of complete activation of the high priority collision avoidance task, a further increase of the stiffness would not have an effect anymore. The direct relation between translational Cartesian error and the penetration of the potential field $\left(d_{i, j}\right.$ hand-hand plot) can be identified here easily. The steady state error of the Cartesian impedance is due to the fact that the controller is basically a PD-controller. Furthermore, the impedance is not designed with a feed-forward term. In the right/top diagram of Fig. 13, the transition can be observed. The measured right arm joint torques $\tau_{\text {meas }}$ (right/bottom) indicate no discontinuities during the transition phase. The original Cartesian impedance torques of the right arm are provided in Fig. 14 (top). Tendentially, the shoulder and upper arm joint torques are higher than the lower arm and wrist joint torques. This is due to the longer lever arm w.r.t. the right TCP. The second diagram in Fig. 14 shows the secondary task torques filtered by the null space projector ${ }^{11}$. Below, their derivatives are depicted and feature the desired boundedness. Beside the expected noise due to the numerical differentiation, peak values lower than $60 \mathrm{Nm} / \mathrm{s}$ can be identified, mainly generated in the first arm joints. The curves from elbow to wrist are omitted here and represented by the shaded rectangle instead. The ratio $r_{\text {sec }}=\left\|\boldsymbol{\tau}_{\text {sec,proj }}\right\| /\left\|\boldsymbol{\tau}_{\text {sec }}\right\|$ at the bottom indicates the actual capability to accomplish the secondary task. While the Cartesian impedance is disturbed in the direction "right hand - left hand", the torques resulting in other directions pass the null space projection unaffectedly. Notice that the peak at $t=2.3 \mathrm{~s}$ is only a side effect of the manner of representation as the torque norms are close to zero and the ratio is very sensitive to variations there.

2) Experiment \#3: In this experiment, the focus is laid on a more complex priority order utilizing (45). This time, the right TCP is commanded into the left hand as illustrated in Fig. 16. After $t=3 \mathrm{~s}$, a continuous trajectory which starts from that

\footnotetext{
${ }^{11}$ Notice that the ordinates of the $\boldsymbol{\tau}_{\text {sec }}$ plot and the $\boldsymbol{\tau}_{\text {sec,proj }}$ plot have the same scaling for better comparison.
}

intermediate TCP location leads back to the initial pose.

Evidently, the intermediate position cannot be reached due to a self-collision and the Cartesian impedance has to be deactivated appropriately to ensure safety. The impedance is projected into the null space of the most critical self-collision avoidance potentials successively. The priority levels are defined by the contact point pairs of the combinations "right hand - left hand" (cpp 1), followed by "right wrist - left hand" (cpp 2) and "right hand - left wrist" (cpp 3). The top plots in Fig. 17 show the activator elements of the three projectors. Although no collision avoidance task is completely activated, all of the repulsive potentials partially disturb the secondary task. Notice that they all "work" in different directions, and therefore, they interfere the impedance multidimensionally. The distances $d_{i, j}$ from the collision model are depicted in the second chart. Due to the parameterization of the self-collision avoidance with $d_{0}=0.1 \mathrm{~m}$, repulsive forces are only generated by the field "right hand - left hand". In this experiment, the repulsion is designed to start in the middle of the transition interval. Thus, the transition begins without a simultaneous collision avoidance intervention. That "overlap" is also illustrated in Fig. 16. The third diagram in Fig. 17 shows the Euclidean norms of the Cartesian impedance, its projection via the three null space projectors, and the measured joint torques of the right arm. Obviously, most of the secondary task commands are filtered after $t=1 \mathrm{~s}$. It is noticeable that the measured torques have a significant offset compared to $\left\|\boldsymbol{\tau}_{\text {sec,proj }}\right\|$. This is due to the gravity compensation. But the resemblance between $\left\|\boldsymbol{\tau}_{\text {sec,proj }}\right\|$ and $\left\|\boldsymbol{\tau}_{\text {meas }}\right\|$ is distinct. The bottom plots give insight into the motion of the active, right TCP. According to the infeasibility of the (secondary) impedance task, a significant steady state error remains in the intermediate configuration between $t=1.5 \mathrm{~s}$ and $t=3 \mathrm{~s}$ that verifies the compliance with the priority order. That deviation from the reference location is also the reason for the slightly increasing $\left\|\boldsymbol{\tau}_{\text {sec,proj }}\right\|$ after $t=1.3 \mathrm{~s}$ since the Cartesian impedance is not completely deactivated.

A supplementary video is attached to this paper which shows: a) several exemplary scenes recorded at a public presentation of the self-collision avoidance, b) a comparison of different damping behaviors, c) reactive self-collision avoidance in a task hierarchy during human-robot interaction, and d) reactive self-collision avoidance in a task hierarchy during autonomous operations.

\section{SUMMARY}

In this paper, a framework for the integration of torquebased self-collision avoidance into a task hierarchy was given. We derived a new algorithm for reactive self-collision avoidance that featured several characteristics beyond the state of the art: A configuration dependent damping enabled the manipulator to systematically dissipate kinetic energy which is crucial if multiple potential collisions are considered. Despite the large number of degrees of freedom, the collision model took all possible collisions into account simultaneously while remaining real-time applicable. As safety features like our self-collision avoidance are described by unilateral constraints, 

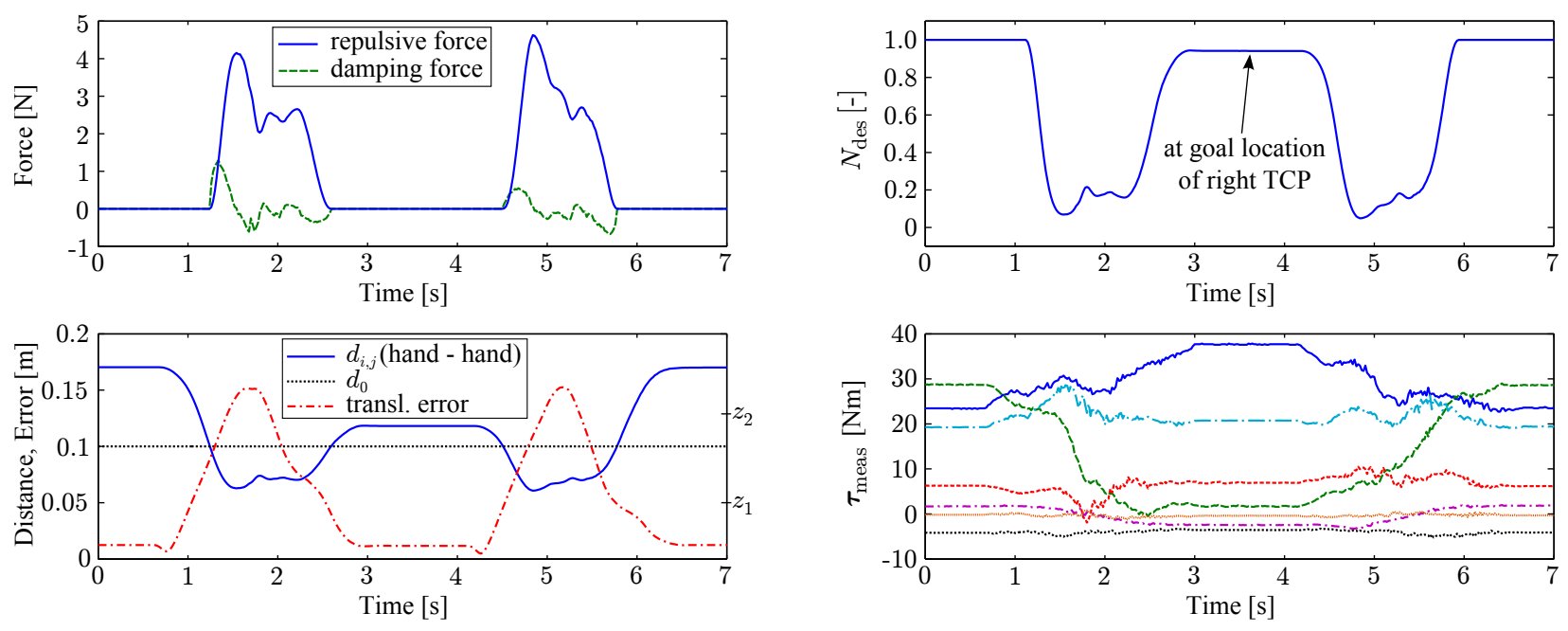

Fig. 13. Experiment \#2: The secondary task is feasible at the goal location of the right TCP. Data about the self-collision avoidance (top priority) and secondary task execution (Cartesian impedance) is given in the left diagrams. The transition behavior is illustrated in the right/top plot. The legend for the distinction of the measured right arm joint torques (right/bottom) is given in Fig. 14 (top).

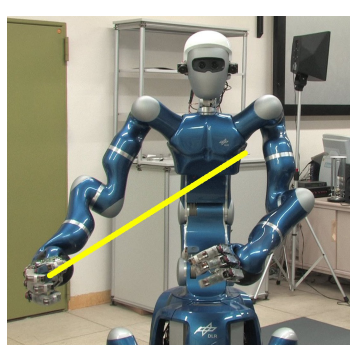

(a) $t=0.7 \mathrm{~s}$

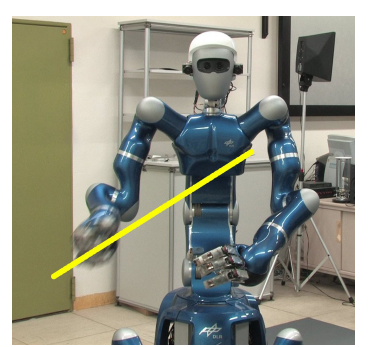

(b) $t=1.2 \mathrm{~s}$

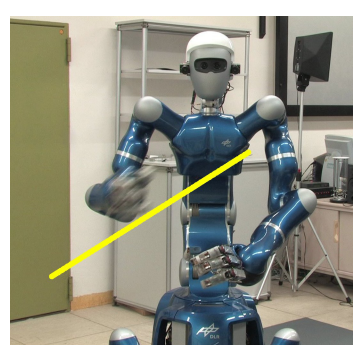

(c) $t=1.7 \mathrm{~s}$

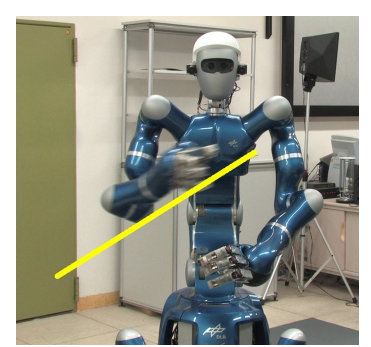

(d) $t=2.2 \mathrm{~s}$

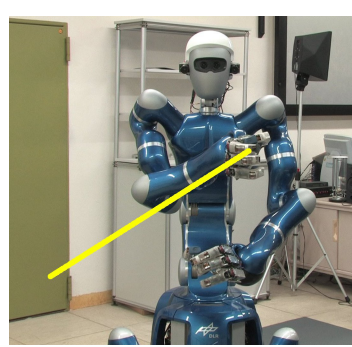

(e) $t=2.7 \mathrm{~s}$

Fig. 15. Snapshots during experiment \#2: Primary task is the self-collision avoidance between the hands. Secondary task is a 6 DOF Cartesian impedance of the right TCP. The trajectory (yellow line) with a total length of $0.8 \mathrm{~m}$ and a maximum translational velocity of $0.6 \mathrm{~m} / \mathrm{s}$ is realized within $2 \mathrm{~s}$.

common redundancy resolution techniques could not be applied to integrate them into a task hierarchy. We extended the well-established, classical null space projection methods to deal with the problems induced by this peculiarity. In a last step, we merged the self-collision avoidance and the redundancy resolution. An implementation of the combined approaches on the multi-DOF humanoid robot Justin of the German Aerospace Center (DLR) was done. Several experiments have been conducted to validate the performance of our synthetic approach. It was shown that our method ensured avoidance of collisions with the own structure while providing a continuous control law and complying with the priority order.

Our redundancy resolution for unilateral constraints is a generic solution and can be applied to arbitrary manipulators and scenarios. While this paper specifically focused on the integration of self-collision avoidance into a task hierarchy, there are no limitations to utilize the redundancy resolution for other unilateral constraints like collision avoidance with external obstacles or further potential field-based tasks. The proposed approach will serve as a fundamental part of a wholebody control concept initiated in [40], [41].

\section{REFERENCES}

[1] M. Grebenstein, A. Albu-Schäffer, T. Bahls, M. Chalon, O. Eiberger, W. Friedl, R. Gruber, S. Haddadin, U. Hagn, R. Haslinger, H. Höppner, S. Jörg, M. Nickl, A. Nothelfer, F. Petit, J. Reill, N. Seitz, T. Wimböck,
S. Wolf, T. Wüsthoff, and G. Hirzinger, "The DLR Hand Arm System," in Proc. of the 2011 IEEE International Conference on Robotics and Automation, May 2011, pp. 3175-3182.

[2] G. Cheng, H. Sang-Ho, J. Morimoto, A. Ude, J. G. Hale, G. Colvin, W. Scroggin, and S. C. Jacobsen, "CB: a humanoid research platform for exploring neuroscience," Advanced Robotics, vol. 21, no. 10, pp. 1097-1114, 2007.

[3] M. Diftler, J. Mehling, M. Abdallah, N. Radford, L. Bridgwater, A. Sanders, R. Askew, D. Linn, J. Yamokoski, F. Permenter, B. Hargrave, R. Platt, R. Savely, and R. Ambrose, "Robonaut 2 - The First Humanoid Robot in Space," in Proc. of the 2011 IEEE International Conference on Robotics and Automation, May 2011, pp. 2178-2183.

[4] H. Iwata and S. Sugano, "Design of Human Symbiotic Robot TWENDYONE," in Proc. of the 2009 IEEE International Conference on Robotics and Automation, May 2009, pp. 580-586.

[5] C. Borst, T. Wimböck, F. Schmidt, M. Fuchs, B. Brunner, F. Zacharias, P. R. Giordano, R. Konietschke, W. Sepp, S. Fuchs, C. Rink, A. AlbuSchäffer, and G. Hirzinger, "Rollin' Justin - Mobile Platform with Variable Base," in Proc. of the 2009 IEEE International Conference on Robotics and Automation, May 2009, pp. 1597-1598.

[6] O. Khatib, "Real-Time Obstacle Avoidance for Manipulators and Mobile Robots," International Journal of Robotics Research, vol. 5, no. 1, pp. 90-98, Spring 1986.

[7] O. Stasse, A. Escande, N. Mansard, S. Miossec, P. Evrard, and A. Kheddar, "Real-Time (Self)-Collision Avoidance Task on a HRP-2 Humanoid Robot," in Proc. of the 2008 IEEE International Conference on Robotics and Automation, May 2008, pp. 3200-3205.

[8] J. Kuffner, K. Nishiwaki, S. Kagami, Y. Kuniyoshi, M. Inaba, and H. Inoue, "Self-Collision Detection and Prevention for Humanoid Robots," in Proc. of the 2002 IEEE International Conference on Robotics and Automation, May 2002, pp. 2265-2270.

[9] A. De Santis, A. Albu-Schäffer, C. Ott, B. Siciliano, and G. Hirzinger, "The skeleton algorithm for self-collision avoidance of a humanoid 

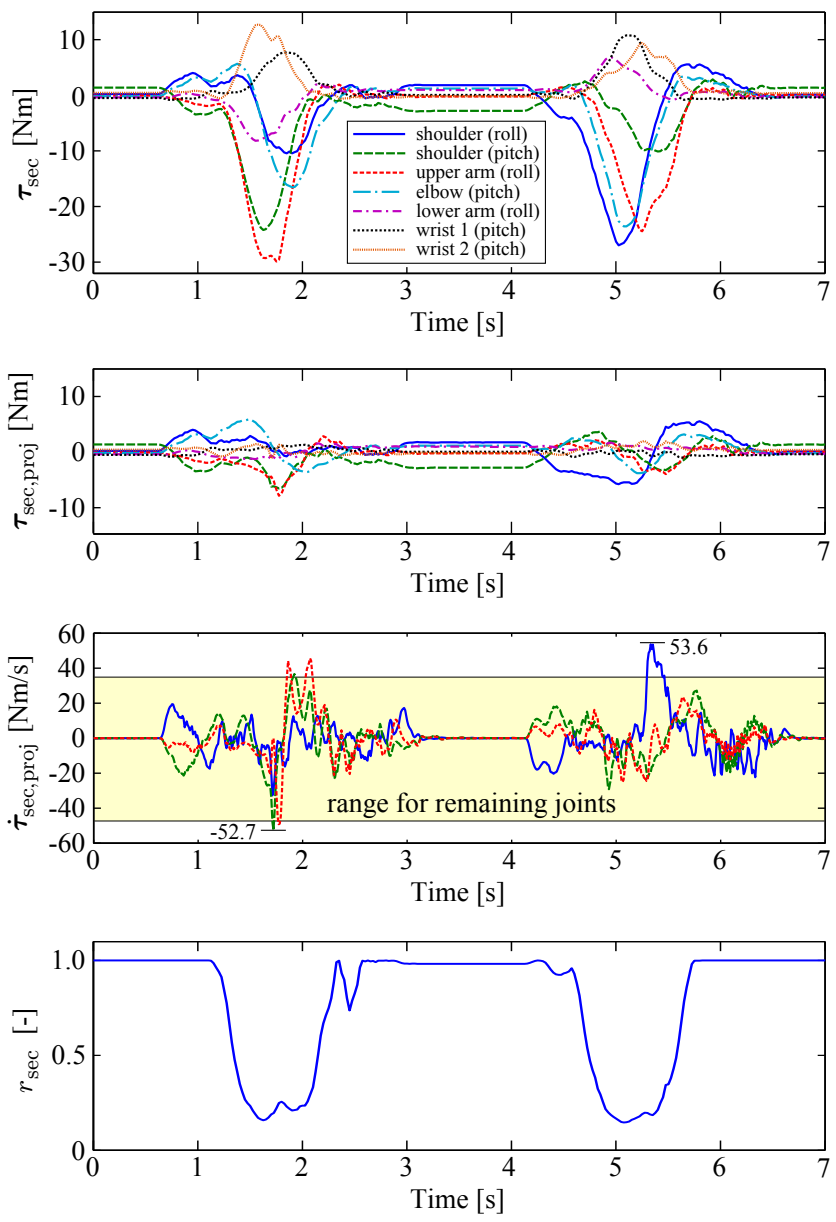

Fig. 14. Experiment \#2: Comparison between original and filtered secondary task torques. For the differentiation, a low-pass filter with $20 \mathrm{~Hz}$ cut-off frequency was applied. The ratio $r_{\text {sec }}=\left\|\boldsymbol{\tau}_{\text {sec,proj }}\right\| /\left\|\boldsymbol{\tau}_{\text {sec }}\right\|$ indicates the ability to execute the secondary task.

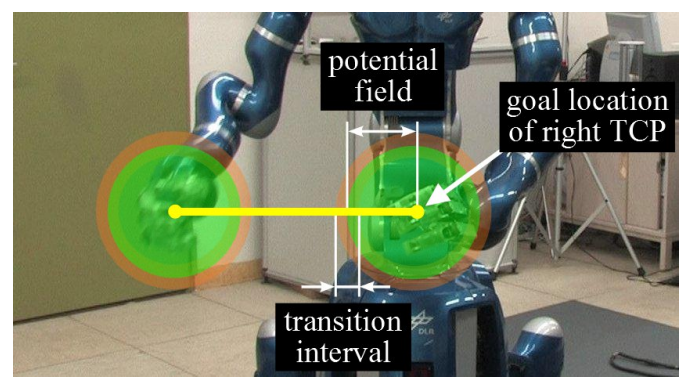

Fig. 16. Experiment \#3: For reasons of clarity, the green circles schematically represent the self-collision avoidance potential fields of all contact point pairs (cpp1, сpp2, and cpp3). In the control, each contact point pair has its own field of course. It can be seen in the figure that the transition interval and the potential field overlap. The latter is a design choice.

manipulator," in Proc. of the 2007 IEEE/ASME International Conference on Advanced Intelligent Mechatronics, September 2007.

[10] S. Haddadin, A. Albu-Schäffer, and G. Hirzinger, "Requirements for Safe Robots: Measurements, Analysis and New Insights," International Journal of Robotics Research, vol. 28, no. 11-12, pp. 1507-1527, Nov./Dec. 2009

[11] H. Sugiura, M. Gienger, H. Janssen, and C. Goerick, "Reactive Self Collision Avoidance with Dynamic Task Prioritization for Humanoid Robots," International Journal of Humanoid Robots, vol. 7, no. 1, pp. 31-54, 2010.

[12] O. Khatib, "A Unified Approach for Motion and Force Control of Robot
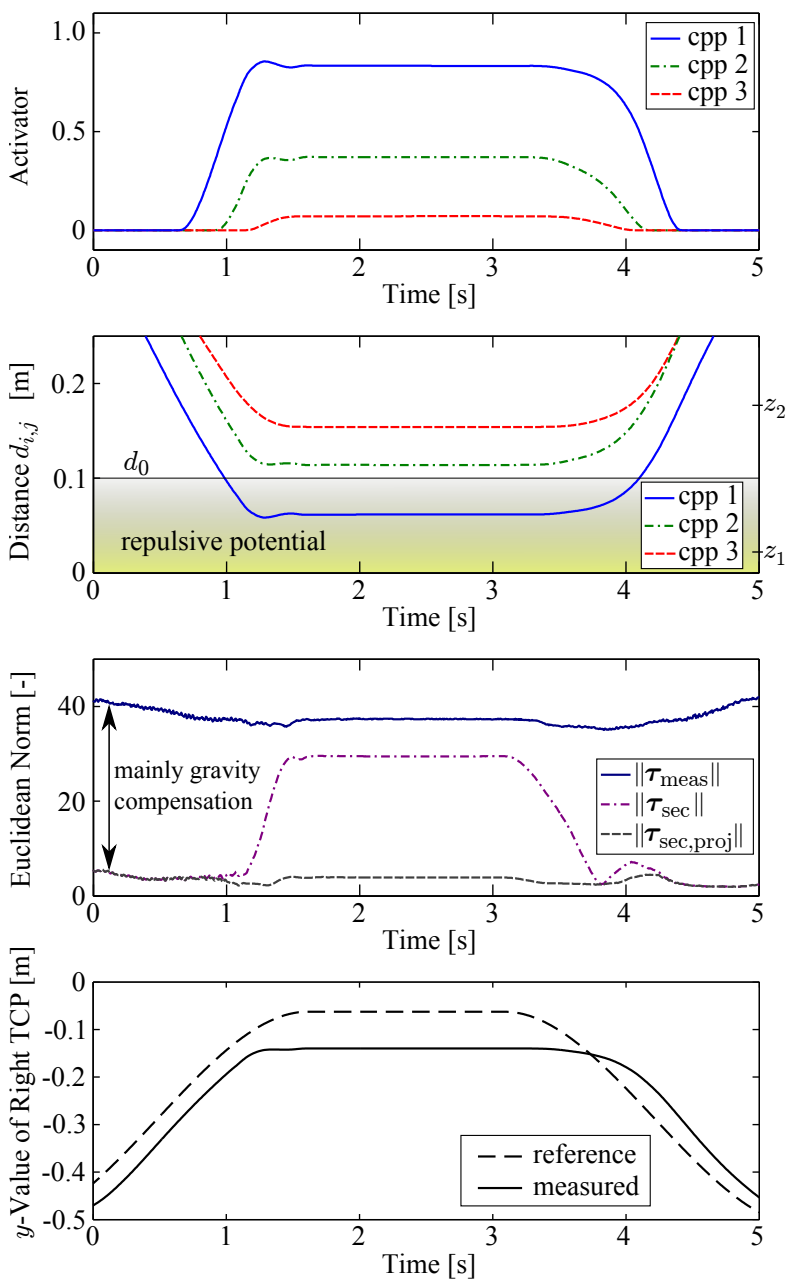

Fig. 17. Experiment \#3: The secondary task (Cartesian impedance) cannot be executed completely due to the activation of the primary task (self-collision avoidance). The contact point pairs (cpp) are defined as follows: cpp 1 ("right hand - left hand"), cpp 2 ("right hand - left wrist"), cpp 3 ("right wrist - left hand").

Manipulators: The Operational Space Formulation," IEEE Journal of Robotics and Automation, vol. RA-3, no. 1, pp. 43-53, February 1987.

[13] Y. Nakamura, H. Hanafusa, and T. Yoshikawa, "Task-Priority Based Redundancy Control of Robot Manipulators," International Journal of Robotics Research, vol. 6, no. 2, pp. 3-15, June 1987.

[14] B. Siciliano and J.-J. Slotine, "A General Framework for Managing Multiple Tasks in Highly Redundant Robotic Systems," in Proc. of the 5th International Conference on Advanced Robotics, June 1991, pp. 1211-1216.

[15] L.-P. Ellekilde and H. I. Christensen, "Control of Mobile Manipulator using the Dynamical Systems Approach," in Proc. of the 2009 IEEE International Conference on Robotics and Automation, May 2009, pp. 1370-1376.

[16] O. Brock, O. Khatib, and S. Viji, "Task-Consistent Obstacle Avoidance and Motion Behavior for Mobile Manipulation," in Proc. of the 2002 IEEE International Conference on Robotics and Automation, May 2002, pp. 388-393.

[17] J. Lee, N. Mansard, and J. Park, "Intermediate Desired Value Approach for Continuous Transition among Multiple Tasks of Robots," in Proc. of the 2011 IEEE International Conference on Robotics and Automation, May 2011, pp. 1276-1282.

[18] N. Mansard, A. Remazeilles, and F. Chaumette, "Continuity of VaryingFeature-Set Control Laws," IEEE Transactions on Automatic Control, vol. 54, no. 11, pp. 2493-2505, November 2009.

[19] N. Mansard, O. Khatib, and A. Kheddar, "A Unified Approach to Integrate Unilateral Constraints in the Stack of Tasks," IEEE Transactions on Robotics, vol. 25, no. 3, pp. 670-685, June 2009. 
[20] S. Chiaverini, "Singularity-Robust Task-Priority Redundancy Resolution for Real-Time Kinematic Control of Robot Manipulators," IEEE Transactions on Robotics and Automation, vol. 13, no. 3, pp. 398-410, June 1997.

[21] J. De Schutter, T. De Laet, J. Rutgeerts, W. Decré, R. Smits, E. Artbeliën K. Claes, and H. Bruyninckx, "Constraint-based Task Specification and Estimation for Sensor-Based Robot Systems in the Presence of Geometric Uncertainty," International Journal of Robotics Research, vol. 26, no. 5, pp. 433-455, May 2007.

[22] W. Decré, R. Smits, H. Bruyninckx, and J. De Schutter, "Extending iTaSC to support inequality constraints and non-instantaneous task specification," in Proc. of the 2009 IEEE International Conference on Robotics and Automation, May 2009, pp. 964-971.

[23] A. Deo and I. Walker, "Overview of Damped Least-Squares Methods for Inverse Kinematics of Robot Manipulators," Journal of Intelligent Robotic Systems, vol. 14, no. 1, pp. 43-68, September 1995.

[24] C. W. Wampler, "Manipulator Inverse Kinematic Solutions Based on Vector Formulations and Damped Least-Squares Methods," IEEE Transactions on Systems, Man, and Cybernetics, vol. 16, no. 1, pp. 93-101, January 1986.

[25] Y. Nakamura and H. Hanafusa, "Inverse Kinematic Solutions With Singularity Robustness for Robot Manipulator Control,' Journal of Dynamic Systems, Measurement, and Control, vol. 108, no. 3, pp. 163171, September 1986.

[26] L. Kelmar and P. Khosla, "Automatic Generation of Kinematics for a Reconfigurable Modular Manipulator System," in Proc. of the 1988 IEEE International Conference on Robotics and Automation, April 1988, pp. 663-668.

[27] P. Baerlocher and R. Boulic, "An Inverse Kinematic Architecture Enforcing an Arbitrary Number of Strict Priority Levels," The Visual Computer, vol. 20, no. 6, pp. 402-417, August 2004.

[28] D. Raunhardt and R. Boulic, "Progressive Clamping," in Proc. of the 2007 IEEE International Conference on Robotics and Automation, April 2007, pp. 4414-4419.

[29] A. Dietrich, T. Wimböck, H. Täubig, A. Albu-Schäffer, and G. Hirzinger, "Extensions to Reactive Self-Collision Avoidance for Torque and Position Controlled Humanoids," in Proc. of the 2011 IEEE International Conference on Robotics and Automation, May 2011, pp. 3455-3462.

[30] A. Dietrich, A. Albu-Schäffer, and G. Hirzinger, "On Continuous Null Space Projections for Torque-Based, Hierarchical, Multi-Objective Manipulation," in Proc. of the 2012 IEEE International Conference on Robotics and Automation, May 2012, pp. 2978-2985.

[31] E. G. Gilbert, D. W. Johnson, and S. S. Keerthi, "A Fast Procedure for Computing the Distance Between Complex Objects in ThreeDimensional Space," IEEE Journal of Robotics and Automation, vol. 4, no. 2, pp. 193-203, April 1988.

[32] R. M. Murray, Z. Li, and S. S. Sastry, A Mathematical Introduction to Robotic Manipulation. CRC Press, 1994.

[33] A. Albu-Schäffer, C. Ott, and G. Hirzinger, "A Passivity Based Cartesian Impedance Controller for Flexible Joint Robots - Part II: Full State Feedback, Impedance Design and Experiments," in Proc. of the 2004 IEEE International Conference on Robotics and Automation, April 2004, pp. 2666-2672.

[34] A. Albu-Schäffer, C. Ott, U. Frese, and G. Hirzinger, "Cartesian Impedance Control of Redundant Robots: Recent Results with the DLR-Light-Weight-Arms," in Proc. of the 2003 IEEE International Conference on Robotics and Automation, Sept. 2003, pp. 3704-3709.

[35] T. F. Chan and R. V. Dubey, "A Weighted Least-Norm Solution Based Scheme for Avoiding Joint Limits for Redundant Joint Manipulators,' IEEE Transactions on Robotics and Automation, vol. 11, no. 2, pp. 286292, April 1995.

[36] C. Ott, Cartesian Impedance Control of Redundant and Flexible-Joint Robots, ser. Springer Tracts in Advanced Robotics. Springer Publishing Company, Berlin Heidelberg, 2008, vol. 49.

[37] L. Sentis and O. Khatib, "Synthesis of Whole-Body Behaviors through Hierarchical Control of Behavioral Primitives," International Journal of Humanoid Robotics, vol. 2, no. 4, pp. 505-518, January 2005.

[38] G. Antonelli, "Stability Analysis for Prioritized Closed-Loop Inverse Kinematic Algorithms for Redundant Robotic Systems," IEEE Transactions on Robotics, vol. 25, no. 5, pp. 985-994, October 2009.

[39] A. Escande, S. Miossec, and A. Kheddar, "Continuous gradient proximity distance for humanoids free-collision optimized-postures," in Proc. of the 7th IEEE/RAS International Conference on Humanoid Robots, Nov.-Dec. 2007, pp. 188-195.

[40] A. Dietrich, T. Wimböck, and A. Albu-Schäffer, "Dynamic Whole-Body Mobile Manipulation with a Torque Controlled Humanoid Robot via Impedance Control Laws," in Proc. of the 2011 IEEE/RSJ International
Conference on Intelligent Robots and Systems, September 2011, pp. 3199-3206.

[41] A. Dietrich, T. Wimböck, A. Albu-Schäffer, and G. Hirzinger, "Reactive Whole-Body Control: Dynamic Mobile Manipulation Using a Large Number of Actuated Degrees of Freedom," IEEE Robotics \& Automation Magazine, vol. 19, no. 2, pp. 20-33, June 2012.

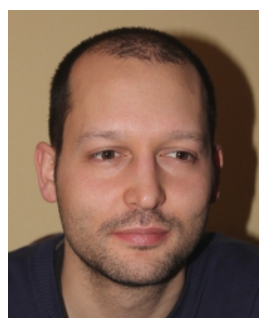

Alexander Dietrich received his Dipl.-Ing. degree in mechanical engineering with focus on control theory and fundamentals in engineering sciences from the Technical University of Munich (TUM) in 2008. In 2010, he joined the German Aerospace Center (DLR), Institute of Robotics and Mechatronics, as a research scientist. His current research interests include impedance and force control, whole-body mobile manipulation, kinematic redundancy, and safe physical human-robot interaction.

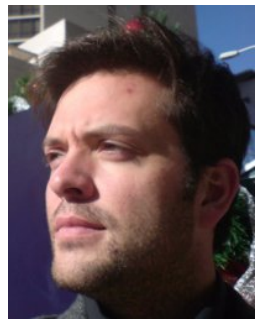

Thomas Wimböck studied electrical engineering at the Rensselaer Polytechnic Institute, Troy, and at the Ecole Polytechnique Fédérale de Lausanne, Switzerland. He received his B.Sc and M.Sc. (in 2004) degreed in electrical engineering from TUM. In 2004, he joined the DLR, Institute of Robotics and Mechatronics, as a research assistant, where he leads the group on dexterous manipulation since 2011. His research interests include nonlinear control, dexterous robot hands, impedance control, VS kinematic redundancy. control, two-handed (humanoid) manipulation, and

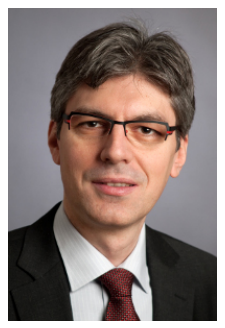

Alin Albu-Schäffer received the Dipl.-Ing. degree in electrical engineering from the Technical University of Timisoara, Romania in 1993 and the Ph.D. degree in control systems from TUM in 2002. Since 1995, he has been working with the DLR, Institute of Robotics and Mechatronics, Germany, where he is currently head of the department for mechatronic components and systems. His current research interests include robot modeling and control, nonlinear control, flexible joint robots, impedance and force control, physical human-robot interaction, and variable impedance actuation.

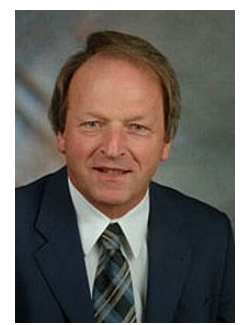

Gerd Hirzinger received the Dipl.-Ing. and Ph.D. degrees from TUM, Germany, in 1969 and 1974, respectively. Since 1992, he has been the director at the DLR, Institute of Robotics and Mechatronics, Wessling, Germany. In 1991, he received a joint professorship from TUM and in 2003, an honorary professorship at the Harbin Institute of Technology, China. He was prime investigator of the first remote control space robot ROTEX, which flew onboard the shuttle Columbia in April 1993. He is the recipient of numerous national and international awards, including the Joseph-Engelsberger-Award for achievements in robotic science in 1994, the Leibniz-Award, in 1995, the Japan Robotics Association (JARA) Award, the Karl-Heinz-Beckurts-Award in 1996, and the IEEE Fellow Award in 1997. In 2004, he received the order of merit of the Federal Republic of Germany and became a member of the Wall of Fame of the Heinz Nixdorf Computer Museum. He is also the recipient of the IEEE Pioneer Award of the Robotics and Automation Society, the 2005 Honorary Citizenship of Budapest Tech, and the IEEE Field Award Robotics and Automation in 2007. 